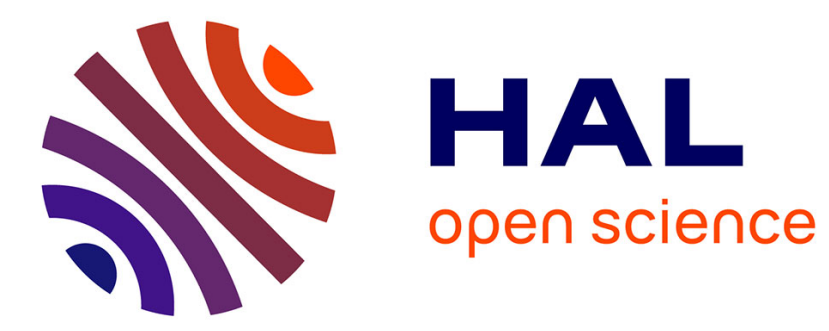

\title{
Transitions between corona, glow, and spark regimes of nanosecond repetitively pulsed discharges in air at atmospheric pressure
}

\author{
David Z. Pai, Deanna A. Lacoste, C. Laux
}

\section{To cite this version:}

David Z. Pai, Deanna A. Lacoste, C. Laux. Transitions between corona, glow, and spark regimes of nanosecond repetitively pulsed discharges in air at atmospheric pressure. Journal of Applied Physics, 2010, 107, pp.093303. 10.1063/1.3309758 . hal-00669396

\section{HAL Id: hal-00669396 https://hal.science/hal-00669396}

Submitted on 13 Feb 2012

HAL is a multi-disciplinary open access archive for the deposit and dissemination of scientific research documents, whether they are published or not. The documents may come from teaching and research institutions in France or abroad, or from public or private research centers.
L'archive ouverte pluridisciplinaire HAL, est destinée au dépôt et à la diffusion de documents scientifiques de niveau recherche, publiés ou non, émanant des établissements d'enseignement et de recherche français ou étrangers, des laboratoires publics ou privés. 


\title{
Transitions between corona, glow, and spark regimes of nanosecond repetitively pulsed discharges in air at atmospheric pressure
}

\author{
David Z. Pai, ${ }^{a}{ }^{2}$ Deanna A. Lacoste, and Christophe O. Laux \\ Laboratoire EM2C, CNRS UPR288, Ecole Centrale Paris, 92295 Châtenay-Malabry, France
}

(Received 18 October 2009; accepted 12 January 2010; published online 6 May 2010)

\begin{abstract}
In atmospheric pressure air preheated from 300 to $1000 \mathrm{~K}$, the nanosecond repetitively pulsed (NRP) method has been used to generate corona, glow, and spark discharges. Experiments have been performed to determine the parameter space (applied voltage, pulse repetition frequency, ambient gas temperature, and interelectrode gap distance) of each discharge regime. In particular, the experimental conditions necessary for the glow regime of NRP discharges have been determined, with the notable result that there exists a minimum and maximum gap distance for its existence at a given ambient gas temperature. The minimum gap distance increases with decreasing gas temperature, whereas the maximum does not vary appreciably. To explain the experimental results, an analytical model is developed to explain the corona-to-glow (C-G) and glow-to-spark (G-S) transitions. The C-G transition is analyzed in terms of the avalanche-to-streamer transition and the breakdown field during the conduction phase following the establishment of a conducting channel across the discharge gap. The G-S transition is determined by the thermal ionization instability, and we show analytically that this transition occurs at a certain reduced electric field for the NRP discharges studied here. This model shows that the electrode geometry plays an important role in the existence of the NRP glow regime at a given gas temperature. We derive a criterion for the existence of the NRP glow regime as a function of the ambient gas temperature, pulse repetition frequency, electrode radius of curvature, and interelectrode gap distance. () 2010 American Institute of Physics. [doi:10.1063/1.3309758]
\end{abstract}

\section{INTRODUCTION}

Atmospheric pressure air plasmas have potential applications in biomedical and surface treatment, chemical and biological decontamination, aerodynamic flow control, and combustion. ${ }^{1-5}$ Many of these applications require diffuse nonthermal (i.e., glow) discharges to meet requirements of large volume, low power, high chemical reactivity, and low gas temperature. At atmospheric pressure, glow discharges in air easily transition into spark discharges that significantly heat the gas, which is problematic for applications sensitive to temperature. ${ }^{2,4,5}$ The methods for preventing the glow-tospark (G-S) transition include inserting resistors or dielectric layers into the discharge circuit. ${ }^{2,6}$ However, such techniques do not optimize the ionization efficiency. The use of highenergy electron beams theoretically optimizes the ionization efficiency ${ }^{7}$ but is hampered by practical challenges at atmospheric pressure.

A practical alternative that is almost as efficient as electron beams for producing glow discharges involves the use of nanosecond repetitively pulsed (NRP) discharges. The application of high-voltage nanosecond-duration pulses generates a strong electric field that accelerates electrons to high energies efficient for ionization. Furthermore, the short pulse duration is chosen to prevent spark formation. Repetitive pulsing results in the accumulation of metastable species important for sustaining the discharge and active species interesting for applications.

\footnotetext{
${ }^{a)}$ Author to whom correspondence should be addressed. Electronic mail: david.pai@em2c.ecp.fr.
}

The first proposal and proof that NRP glow discharges could be produced in air at atmospheric pressure came in air preheated to $2000 \mathrm{~K}^{8-10}$ Attempts were then made to generate the NRP glow discharge at room temperature without success. It was suspected that the long electron recombination time in air at such a high temperature compared to that at room temperature made the generation of glow discharges much easier, owing to the high level of preionization in the channel. Subsequently, the NRP glow regime was studied in preheated air at atmospheric pressure at an ambient gas temperature $\left(T_{0}\right)$ of $1000 \mathrm{~K}$, a pulse repetition frequency $(f)$ of $30 \mathrm{kHz}$, and an interelectrode distance $(d)$ of $5 \mathrm{~mm} \cdot{ }^{11-13}$ By fixing the operating conditions, the properties unique to this regime were isolated and studied. A discussion on how NRP discharges differ from other nanosecond pulsed discharges reported in the literature can be found in Ref. 12.

In this paper, we seek to characterize the dependence of the properties of the NRP glow and spark regimes on $T_{0}, f$, and $d$. This extends the study of discharge regime properties begun in Ref. 12 and it also provides insight on how to choose experimental parameters to obtain a given discharge regime. In other words, we seek to understand the physics of regime transitions for NRP discharges, with a particular emphasis on the necessary conditions for obtaining the glow regime. Thus, we deal primarily with the corona-to-glow (C-G) and G-S transitions. For given experimental conditions, these transitions occur at voltages $V_{\mathrm{CG}}$ and $V_{\mathrm{GS}}$, respectively.

This paper is organized as follows. Sec. II presents the experimental setup. Section III presents experimental results 


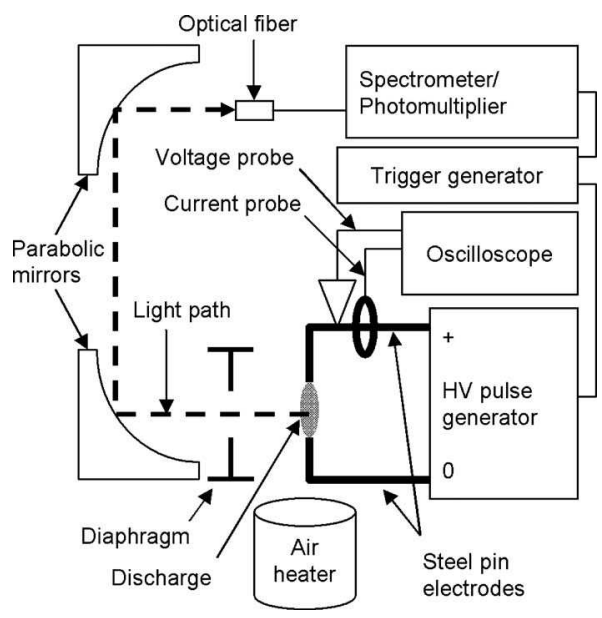

FIG. 1. Schematic diagram of the experimental setup.

on regime transitions of NRP glow and spark discharges. Section III A discusses how the pulse repetition frequency affects the regimes, the emission intensity at different points along the electrode axis, and the energy deposited in the discharge per pulse. Section III B discusses how the ambient gas temperature affects the regimes, the emission intensity, and the energy deposited in the discharge per pulse. In Sec. III C, we discuss the influence of the gap distance on the discharge regimes. In Sec. III D we discuss the coupled effect when both the gap distance and the ambient gas temperature are varied. This permits us to choose the operating conditions to generate the NRP glow regime down to $T_{0}$ $=300 \mathrm{~K}$. In Secs. IV A and IV B we present a theoretical framework for understanding the C-G and G-S transitions, respectively. This theory is used in Sec. IV C to derive a criterion for the existence of the NRP glow regime.

\section{EXPERIMENTAL SETUP AND METHODS}

\section{A. Experimental setup}

Figure 1 shows a diagram of the experimental setup for plasma generation and measurement. NRP discharges are produced by short-duration (10 ns) high-voltage $(5-10 \mathrm{kV})$ positive-polarity pulses at pulse repetition frequencies $(f)$ of $1-30 \mathrm{kHz}$ across a discharge gap $(0.5-10 \mathrm{~mm})$ formed by two steel electrodes in a vertical pin-pin configuration. The NRP discharges are created in preheated $(300-1000 \mathrm{~K})$ atmospheric pressure air flowing at $0.5-2.6 \mathrm{~m} / \mathrm{s}$.

The discharge is generated in an airflow preheated to $300-1000 \mathrm{~K}$ by a resistive heater (Osram Sylvania $8 \mathrm{~kW}$ Hot Air System). A digital mass flow meter calibrated for air regulates $5 \mathrm{~atm}$ compressed air down to $1 \mathrm{~atm}$, which then flows into the resistive heater where it passes through a ceramic heating coil. An internal thermocouple located $2.5 \mathrm{~cm}$ upstream of the heater exit measures the heated air temperature to feed the temperature regulation control loop. Separate thermocouple measurements at about $2 \mathrm{~cm}$ downstream of the heater exit, where the discharge gap is located, confirmed that the temperature remained stable within $50{ }^{\circ} \mathrm{C}$ of the heater's internal thermocouple reading over run times of several hours.
The plasma discharge is produced by applying nanosecond high-voltage pulses between two steel electrodes, as shown in Fig. 2. The majority of the results reported in this work have been obtained using an FID Technologies generator (Model FPG 10-30MS) that produces pulses with rise/ fall times of $5 \mathrm{~ns}$, a flat top of $10 \mathrm{~ns}$ at greater than $90 \%$ of the maximum voltage, and amplitudes up to $+5 \mathrm{kV}$ into a matching $75 \Omega$ impedance, or up to $+10 \mathrm{kV}$ into an open circuit. The pulse repetition frequency $(f)$ can be varied up to $30 \mathrm{kHz}$. Some experiments have been performed using another FID Technologies generator (Model FPG 30$100 \mathrm{MC} 4 \mathrm{~K})$ that produces pulses with rise/fall times of $5 \mathrm{~ns}$, a flat top of $10 \mathrm{~ns}$ at greater than $90 \%$ of the maximum voltage, and amplitudes up to $+30 \mathrm{kV}$ into a matching $300 \Omega$ impedance, or up to $+60 \mathrm{kV}$ into an open circuit, with $f=0-100 \mathrm{kHz}$. In the following, results obtained using the FPG $30-100 \mathrm{MC} 4 \mathrm{~K}$ will be indicated as such. The electrodes are placed in a vertical pin-pin configuration with the anode above the grounded cathode.

The voltage across the electrodes is measured with a 100-MHz bandwidth high-voltage probe (Lecroy PPE20kV). The response of the PPE20kV to pulsed signals with rise times and durations similar to those of the high-voltage pulses applied across the electrodes has been validated by comparison to the responses of a PMK PHV641-L highvoltage probe and a $2 \mathrm{~m}$ long RG58C/U coaxial cable, whose respective bandwidths are $380 \mathrm{MHz}$ and $1 \mathrm{GHz}{ }^{11}$ The discharge current is measured with a Pearson coil current monitor (Model 6585) with a $3 \mathrm{~dB}$ cutoff low frequency of 400 $\mathrm{Hz}, \mathrm{a} \pm 3 \mathrm{~dB}$ high frequency of $250 \mathrm{MHz}$, a $1.5 \mathrm{~ns}$ rise time, and a $1 \mathrm{~A} / 1 \mathrm{~V}$ current-to-voltage conversion when terminated with a $1 \mathrm{M} \Omega$ impedance. A low-voltage attenuator (Barth Model 2-20), not shown in Fig. 2, with $20 \mathrm{~dB}(10 \times)$ attenuation and $0-20 \mathrm{GHz}$ bandwidth immediately follows the current monitor. The probe signals are recorded with a $350 \mathrm{MHz}$ (maximum 1 or $2 \mathrm{GS} / \mathrm{s}$ ) LeCroy Waverunner 434 or a $1 \mathrm{GHz}$ (maximum 5 or $10 \mathrm{GS} / \mathrm{s}$ ) LeCroy Wavepro 7000 oscilloscope. Unless indicated otherwise, all measurements presented in this paper are averaged over 100 samples.

For optical emission spectroscopy, two $90^{\circ}$ off-axis parabolic UV-grade $\mathrm{MgF}_{2}$-coated aluminum mirrors of $50 \mathrm{~mm}$ diameter (Janos Technologies) collect and focus light into a UV solarization-resistant optical fiber (Ocean Optics QP6002-SR-BX). A diaphragm placed before the collecting mirror is used to adjust the solid angle to control the intensity of the light collected, and a pinhole is placed before the optical fiber to ensure spatial resolution. To isolate a specific transition for photomultiplier tube (PMT) measurements, a bandpass interference filter [e.g., Melles Griot 03 FIU 127 for the $\mathrm{N}_{2}$ (C-B) 0-0 transition] is inserted into the optical path. The light collection system is placed on a $20 \times 25 \mathrm{~cm}^{2}$ optical table with X-Z translation capability.

Spectral measurements are made with a spectrometer consisting of a monochromator (Acton SpectraPro 2500i) fitted with an intensified charge coupled device (ICCD) camera (Princeton Instruments PI-MAX). For time-resolved emission intensity measurements, a PMT with a 1.4 ns rise time (Hamamatsu H9305-3) is used in place of the spectrometer. 


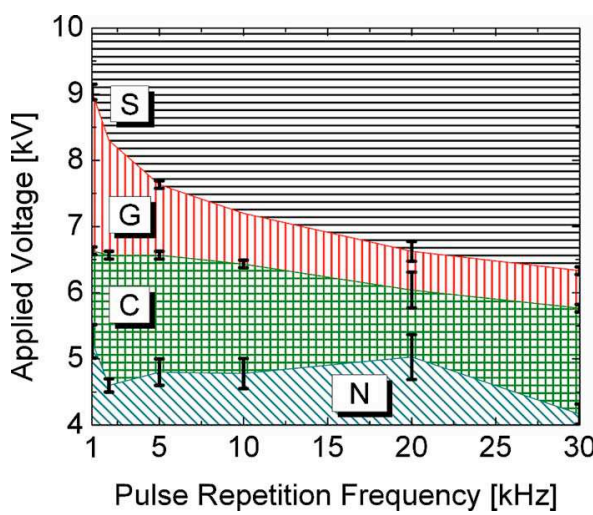

FIG. 2. (Color online) Discharge regime map according to applied voltage $\left(V_{\mathrm{p}}\right)$ as a function of pulse repetition frequency $(f) . T_{0}=1000 \mathrm{~K}, d$ $=5 \mathrm{~mm}$, and $v=1.6 \mathrm{~m} / \mathrm{s}$. S: Spark, G: Glow, C: Corona, and N: No discharge.

The PMT signal is amplified using a $2 \mathrm{GHz}$ high-speed amplifier (Hamamatsu C5594-44) and then recorded using the oscilloscope.

\section{B. Method for measuring the energy deposited per pulse}

In Secs. III A and III B, we will discuss measurements of the energy per pulse deposited by the discharge. As the energies can be quite small, it is necessary to consider carefully the method of measurement.

To obtain the energy, we measured the applied voltage and the discharge current using the Lecroy PPE20kV highvoltage probe and the Pearson Model 6585 current probe, respectively. We multiply the measured voltage and current waveforms to obtain the power, then integrate in time over the pulse duration $\left(\tau_{\mathrm{p}}\right)$ to yield the energy. Our intention is to study the action of well-defined pulses at regular intervals, and therefore we do not consider nonideal power dissipation through oscillations and secondary pulses produced by the generator. The total measured current includes the conduction, displacement, and parasitic currents. The parasitic current is mainly due to the stray capacitance of the $20 \mathrm{~cm}$ coaxial cable connecting the generator to the electrodes. We assume that the energy associated with the displacement current is zero over the integration period. Thus, the measured total energy per pulse is the sum of the energy dissipated into the plasma and the parasitic energy loss.

As discussed in Refs. 11 and 12 the conduction current for NRP glow discharges is very small, and the voltage and current waveforms are nearly indistinguishable from the corresponding waveforms when no plasma is present, i.e., when the high-voltage pulse generator is operated using the same settings but in ambient air such that no discharge is created. However, there is a measurable difference in energy deposited per pulse between the plasma and no-plasma cases. Without plasma, no conduction current flows, and the measured energy reflects only parasitic losses. We verified that the measured energy per pulse is always greater with the discharge than without it. ${ }^{11}$ Thus, the energy deposited by the
NRP glow discharge is taken to be the difference between the measurements with the discharge and those without discharge.

For the spark regime, the energy measured with the discharge is always measured to be about ten times greater than that of the corresponding case without discharge, and therefore it is not necessary to correct the measurement of energy with discharge.

\section{EXPERIMENTAL RESULTS}

We assembled the measured transition voltage data into "maps" of the discharge regimes as a function of experimental parameters such as the pulse repetition frequency $(f)$, the gas temperature, and the interelectrode gap distance. The corona regime is lower-bounded by the corona onset voltage $\left(V_{\mathrm{NC}}\right)$ and upper-bounded by the C-G transition voltage $\left(V_{\mathrm{CG}}\right)$. The glow regime is lower-bounded by $V_{\mathrm{CG}}$ and upperbounded by the G-S transition voltage $\left(V_{\mathrm{GS}}\right)$, and the spark regime is lower-bounded by $V_{\mathrm{GS}}$, with no upper bound found up to $10 \mathrm{kV}$, the maximum output of the high-voltage pulse generator. The regimes are sensitive to the sharpness of the electrode tips and their surface condition. In particular, exposure to the spark regime gradually erodes the cathode, leading to higher voltages for regime transitions. For consistency, the electrodes were sharpened and cleaned as often as possible. In addition, we stopped operating the high-voltage pulse generator immediately after the spark regime was attained to minimize electrode damage.

\section{A. Influence of the pulse repetition frequency on discharge regimes}

In this section we present measurements of the applied voltage $\left(V_{\mathrm{p}}\right)$ ranges over which NRP discharge regimes occur, as a function of the pulse repetition frequency $(f)$. For all of these measurements, we fixed the temperature of the flowing air at $T_{0}=1000 \mathrm{~K}$. For each $f$ under study, we started from $V_{\mathrm{p}}=0$ and measured the voltages for the corona onset, the C-G transition, and the G-S transition using the Lecroy PPE20kV high-voltage probe. Figure 2 shows a map of the discharge regimes as a function of $f$ with fixed $T_{0}$ $=1000 \mathrm{~K}, d=5 \mathrm{~mm}$, and $v=1.6 \mathrm{~m} / \mathrm{s}$. $V_{\mathrm{NC}}, V_{\mathrm{CG}}$, and $V_{\mathrm{GS}}$ were recorded for $f=1-30 \mathrm{kHz}$.

The limits of the regimes do not show much variation with $f$, except for the glow regime, whose applied voltage range expands at low $f$. In the corona regime, the emission zone in the anode region progressively enlarges across the discharge gap as the applied voltage increases, until it fully connects with the cathode, thus transitioning to the glow regime. The luminosity of the discharge jumps noticeably at the transition. The G-S transition is also distinct, both visibly and audibly, as the luminosity greatly increases and a crackling sound appears. Both the C-G and G-S transitions usually occur abruptly when the applied voltage is increased. At high $f$, the regime transitions are very distinct visually. At low $f$, however, the average luminosity of the discharge is low, requiring the use of the spectrometer to monitor the plasma emission. 


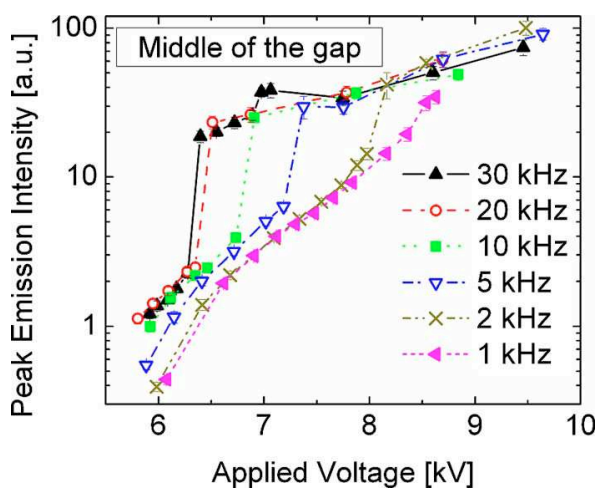

FIG. 3. (Color online) Measured peak emission intensity of $\mathrm{N}_{2} \mathrm{C}-\mathrm{B}(0,0)$ in the middle of the discharge gap as a function of $V_{\mathrm{p}}$ for $f=1-30 \mathrm{kHz} . T_{0}$ $=1000 \mathrm{~K}, d=5 \mathrm{~mm}$, and $v=1.6 \mathrm{~m} / \mathrm{s}$.

Figure 3 shows the peak emission intensity of the $(0,0)$ band of the $\mathrm{N}_{2}$ (C-B) transition at the center of the discharge gap for the glow and spark regimes, as a function of the applied voltage for $f=1-30 \mathrm{kHz}$, with $T_{0}=1000 \mathrm{~K}, d$ $=5 \mathrm{~mm}$, and $v=1.6 \mathrm{~m} / \mathrm{s}$ fixed. Comparing with the regime map presented in Fig. 2, we find that the jumps in emission correspond to the G-S transition at each $f$. These jumps are about a factor of 10 at high $f$ but gradually reduce until there is virtually no jump at $f=1 \mathrm{kHz}$. Thus, the G-S transition becomes "softer" with decreasing $f$. Within each regime, there are up to two decades of variation in the peak emission intensity, and thus there is a likewise variation in the density of the excited electronic states of $\mathrm{N}_{2}$. The corresponding variation in applied voltage is less than $50 \%$, and therefore there is a considerable margin for tuning the plasma chemistry for applications that are insensitive to applied voltage.

Furthermore, Fig. 2 shows that the applied voltage range corresponding to the glow regime expands with decreasing $f$. We see from Fig. 3 that the range of emission of the glow regime increases in similar fashion with decreasing $f$. By using lower $f$, we can obtain glow discharges that emit at intensities above the range attainable at higher $f$. This effect may present a simple way to tune the emission intensity, and by extension the plasma chemical activity, for applications that are insensitive to the pulse repetition frequency. Overall, the emission intensity in the glow regime is more sensitive to changes in the applied voltage $\left(V_{\mathrm{p}}\right)$ than in $f$, and in the spark regime the emission varies slowly with $f$.

If we interpret the emission intensity of the $\mathrm{N}_{2}$ (C-B) $(0,0)$ transition as a general indicator of the chemical activity of the plasma, then we see that $f$ does not so much affect the chemistry of the glow regime as it determines the G-S transition voltage. In contrast, the applied voltage has a strong effect on the degree of chemical activity. Thus, the accumulative effect of repetitive pulsing on the chemistry is not as significant as the influence of the applied electric field, within the range of pulse repetition frequencies and applied voltages studied here.

Figure 4 shows the measured energies per pulse of the glow and spark regimes as a function of the applied voltage for $f=1-30 \mathrm{kHz}$, with $T_{0}=1000 \mathrm{~K}, d=5 \mathrm{~mm}$, and $v$ $=1.6 \mathrm{~m} / \mathrm{s}$ held constant. The G-S transition is marked by an abrupt increase in energy that grows with increasing $f$, from

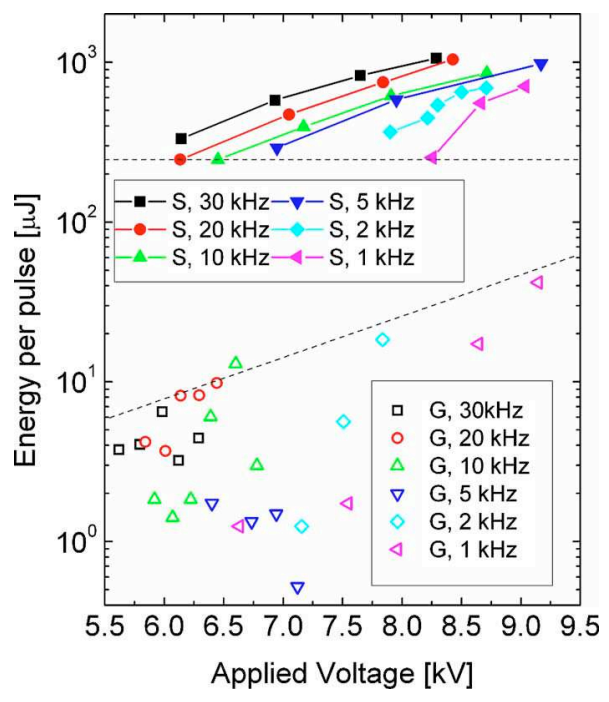

FIG. 4. (Color online) Measured energy per pulse for the glow (hollow) and spark (solid) regimes as a function of applied voltage for $f=1-30 \mathrm{kHz}$. $T_{0}=1000 \mathrm{~K}, d=5 \mathrm{~mm}$, and $v=1.6 \mathrm{~m} / \mathrm{s}$.

a factor of 6 to 50. Another important feature is that the energy curves shown for the spark regime shift downward in applied voltage with increasing $f$. This shift also seems to occur for the glow regime, although the trend is not as clear because the energies are much smaller and the measurement uncertainty is higher. Furthermore, the dashed lines in Fig. 4 show that the maximum energy for the glow regime at a given frequency increases with decreasing pulse repetition frequency, whereas the minimum energy for the spark regime remains about the same. Overall, the energy deposited per pulse is about $1-20 \mu \mathrm{J}$ for the glow regime and about 200-1000 $\mu \mathrm{J}$ for the spark regime.

According to Fig. 4, it might be thought that the glow and spark regimes can sometimes occur simultaneously over a range of applied voltages. However, when we increased the applied voltage from $0 \mathrm{~V}$, we always observed that the glow transitioned into the spark without any sustained period of both regimes occurring simultaneously. In reality, for two separate experiments with different electrode conditions, the same applied voltage can generate a glow discharge in one experiment and a spark discharge in the other. As mentioned previously, spark regime experiments progressively erode the electrodes, resulting in changes in $V_{\mathrm{GS}}$. Thus, the overlapping of the regimes shown in Fig. 4 is indicative of changes in $V_{\mathrm{GS}}$, not the coexistence of the glow and spark regimes. To minimize the effects of electrode erosion, we performed all of the glow regime measurements before proceeding to the spark regime measurements.

\section{B. Influence of ambient gas temperature on discharge regimes}

We now apply the same analysis as in Sec. III A to determine the influence of the ambient gas temperature $T_{0}$ on the discharge regimes. Figure 5 presents the regime map as a function of $T_{0}$, with $f=30 \mathrm{kHz}, d=5 \mathrm{~mm}$, and $v=1.6 \mathrm{~m} / \mathrm{s}$ fixed.

The regime map in Fig. 5 shows a much more pronounced dependence on the ambient gas temperature than 


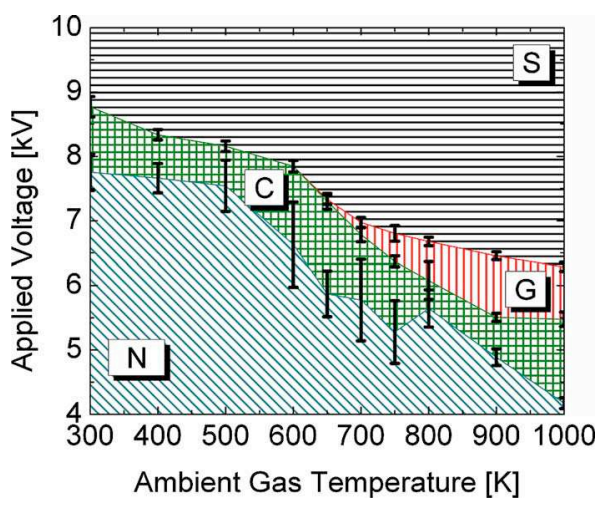

FIG. 5. (Color online) Map of discharge regimes according to applied voltage as a function of ambient gas temperature. $f=30 \mathrm{kHz}, d=5 \mathrm{~mm}$, and $v=1.6 \mathrm{~m} / \mathrm{s}$. S: Spark, G: Glow, C: Corona, and N: No discharge.

did Fig. 2 for $f$. It can be seen that for gas temperatures between 300 and $700 \pm 50 \mathrm{~K}$, only the corona and spark regimes are observed. The glow regime appears only above $700 \pm 50 \mathrm{~K}$ and expands with increasing gas temperature. Thus high temperature conditions are favorable to the creation of the glow regime.

Figure 6 shows the peak emission intensity of $\mathrm{N}_{2}$ (C-B) $(0,0)$ in the glow and spark regimes as a function of the applied voltage at the middle of the discharge gap for $T_{0}$ $=300-1000 \mathrm{~K}$, with $f=30 \mathrm{kHz}, d=5 \mathrm{~mm}$, and $v$ $=1.6 \mathrm{~m} / \mathrm{s}$ fixed. Comparing with the regime map presented in Fig. 5, we see that the jumps in emission correspond to the G-S transition. Unlike the case with varying $f$, the jumps are always by about a factor of 10 . Also, the glow regime does not exist below $750 \mathrm{~K}$ for these experimental conditions, and thus only the emission for the spark regime is available for the 300 and $500 \mathrm{~K}$ cases.

Figure 7 shows the measured energies per pulse of the glow and spark regimes as a function of applied voltage for $T_{0}=500-1000 \mathrm{~K}$, with $f=30 \mathrm{kHz}, d=5 \mathrm{~mm}$, and $v$ $=1.6 \mathrm{~m} / \mathrm{s}$ fixed. The G-S transition is marked by an abrupt increase in energy that grows with increasing $T_{0}$, from a factor of 14 at $700 \mathrm{~K}$ to 32 at $1000 \mathrm{~K}$. Another important feature is that the energy curves shown for the spark regime shift downward in applied voltage with increasing $T_{0}$. This shift also seems to occur for the glow regime, although the trend is not as clear because the energies are much smaller

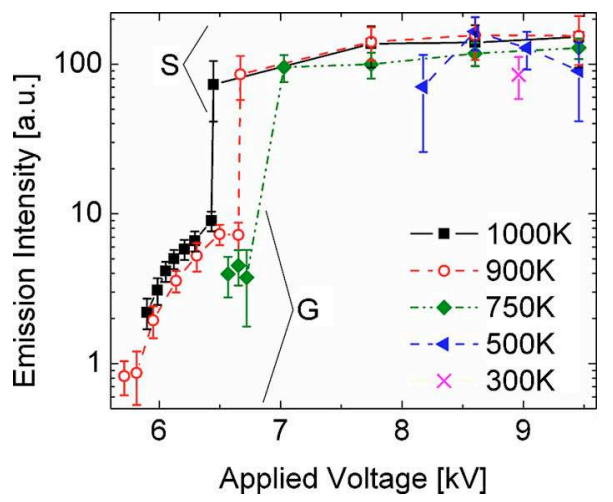

FIG. 6. (Color online) Measured $\mathrm{N}_{2} \mathrm{C}-\mathrm{B}(0,0)$ peak emission intensity in the middle of the discharge gap as a function of the applied voltage for $T_{0}$ $=300-1000 \mathrm{~K} . f=30 \mathrm{kHz}, d=5 \mathrm{~mm}$, and $v=1.6 \mathrm{~m} / \mathrm{s}$.

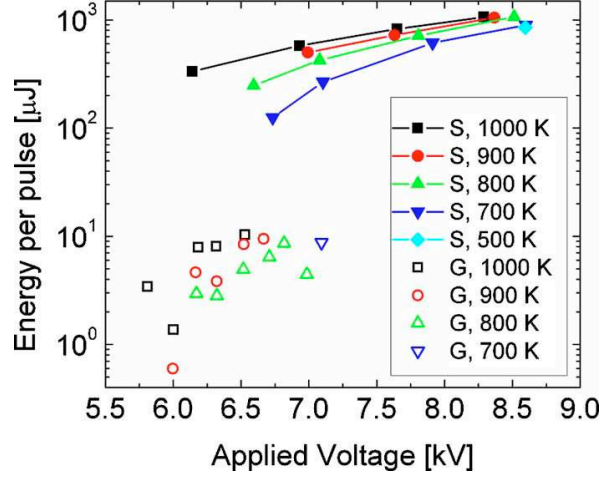

FIG. 7. (Color online) Measured energy per pulse for the glow (hollow) and spark (solid) regimes as a function of the applied voltage for $T_{0}$ $=500-1000 \mathrm{~K} . f=30 \mathrm{kHz}, d=5 \mathrm{~mm}$, and $v=1.6 \mathrm{~m} / \mathrm{s}$.

and the measurement uncertainty is higher. Overall, the energy deposited per pulse is about $1-10 \mu \mathrm{J}$ for the glow regime and about 100-1000 $\mu \mathrm{J}$ for the spark regime.

Figure 7 also shows that the maximum energy for the glow regime remains about constant with $T_{0}$, an observation that will become important in Sec. IV B for explaining the NRP G-S transition. Similar results have been reported for transient glow discharges in air at 300-500 Torr, ${ }^{14}$ for which the glow-to-arc transition occurs when a certain amount of energy is dissipated in the discharge gap during the glow phase. Also, for the glow-to-arc transition in transient glow discharges in air at 1-20 Torr, the density of energy deposited in the cathode region during the glow phase is independent of the gas pressure. ${ }^{15}$

\section{Influence of interelectrode gap distance on discharge regimes}

Experiments were also performed to construct a regime map as a function of the gap distance. Figure 8 presents the regime map in applied voltage as a function of gap distance with $T_{0}=1000 \mathrm{~K}, f=30 \mathrm{kHz}$, and $v=1.6 \mathrm{~m} / \mathrm{s}$ fixed. Under $d=3 \mathrm{~mm}$ only the spark regime exists. Thus there is a minimum gap distance $d_{\min }=3 \mathrm{~mm}$ above which the corona and glow regimes can occur, as well as a maximum gap distance $d_{\max }=9 \mathrm{~mm}$ above which the glow no longer occurs.

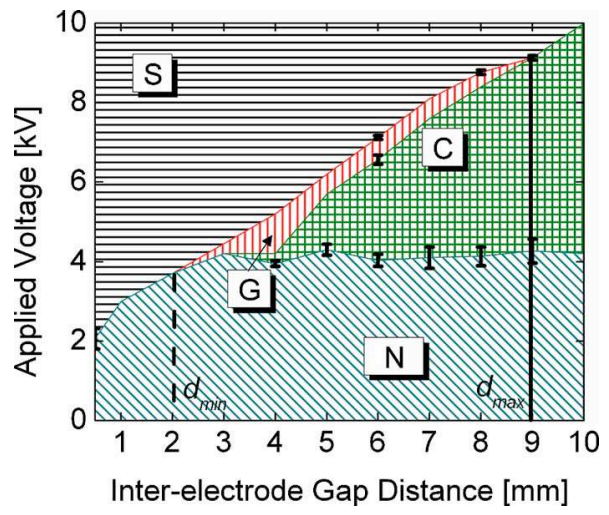

FIG. 8. (Color online) Discharge regime map according to applied voltage as a function of gap distance. $T_{0}=1000 \mathrm{~K}, f=30 \mathrm{kHz}$, and $v=1.6 \mathrm{~m} / \mathrm{s}$. S: Spark, G: Glow, C: Corona, and N: No discharge. 


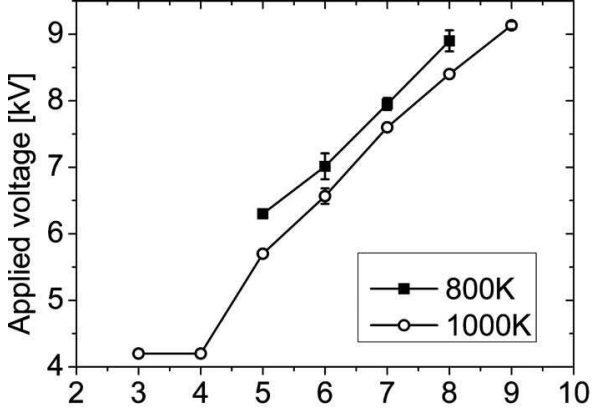

(a) Inter-electrode gap distance [mm]

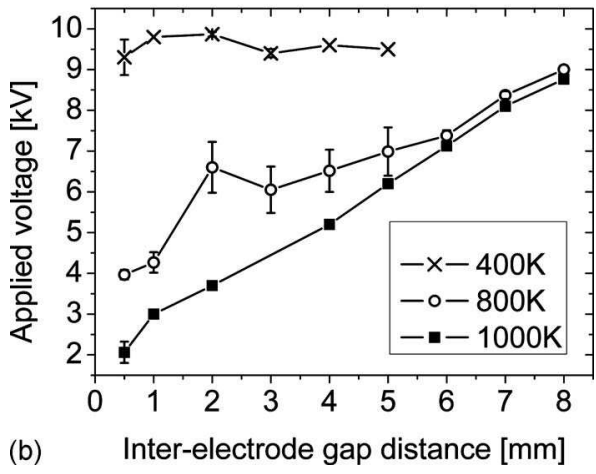

FIG. 9. (a) Measured C-G transition voltage as a function of $d$ for different $T_{0}$, and (b) measured voltage for the transition to spark (N-S, C-S, and G-S) as a function of $d$ for different $T_{0} . f=30 \mathrm{kHz}$, and $v=1.6 \mathrm{~m} / \mathrm{s}$.

In Fig. 8, the glow regime occupies a thin region centered about a line segment whose slope is approximately 10 $\mathrm{kV} / \mathrm{cm}$. This value corresponds to the electric field for breakdown at $1 \mathrm{~atm}$ and $T_{0}=1000 \mathrm{~K} \cdot{ }^{16}$ For a glow discharge, the average electric field in the positive column $\left(E_{\mathrm{pc}}\right)$ is equal to $\left(V_{\mathrm{p}}-V_{\mathrm{cf}}\right) / d$, where $V_{\mathrm{cf}}$ is the cathode fall voltage. The positive column length is approximately the gap distance because the anode and cathode fall regions of a glow discharge are typically only microns thick for atmospheric pressure air. ${ }^{16}$ Thus, using a linear fit of the applied voltage $V_{\mathrm{p}}$ as a function of gap distance, we find that $E_{\mathrm{pc}}$ corresponds to the slope of the line segment and $V_{\text {cf }}$ is the vertical intercept at $d=0$. For the conditions of Fig. 8, for example, we find $E_{\mathrm{pc}}$ $=10 \mathrm{kV} / \mathrm{cm}$ and $V_{\mathrm{cf}}=2 \mathrm{kV}$. We will consider specific values of $E_{\mathrm{pc}}$ and $V_{\mathrm{cf}}$ in Sec. IV A 2.

Figure 9(a) shows the $\mathrm{C}-\mathrm{G}$ transition voltages as a function of gap distance for $T_{0}=800$ and $1000 \mathrm{~K}$. The data for $1000 \mathrm{~K}$ are taken from Fig. 8. We see again that $V_{\mathrm{CG}}$ depends linearly on the gap distance. Furthermore, we have the surprising result that the minimum distance for the existence of the glow regime increases as the temperature decreases, going from $3 \mathrm{~mm}$ at $1000 \mathrm{~K}$ to $5 \mathrm{~mm}$ at $800 \mathrm{~K}$.

Figure 9(b) shows the voltages for transition to spark as a function of the gap distance for 400,800 , and $1000 \mathrm{~K}$. Note that this includes transitions to spark from the corona and glow regimes, as well as from no discharge whatsoever. The transition voltages increase with decreasing gas temperature. Furthermore, note that the transition to spark at $1000 \mathrm{~K}$ shown in Fig. 9(b) does not simply shift to higher applied voltage at $800 \mathrm{~K}$, in contrast to the behavior of the $\mathrm{C}-\mathrm{G}$ transition shown in Fig. 9(a). In Sec. IV C, we will show that

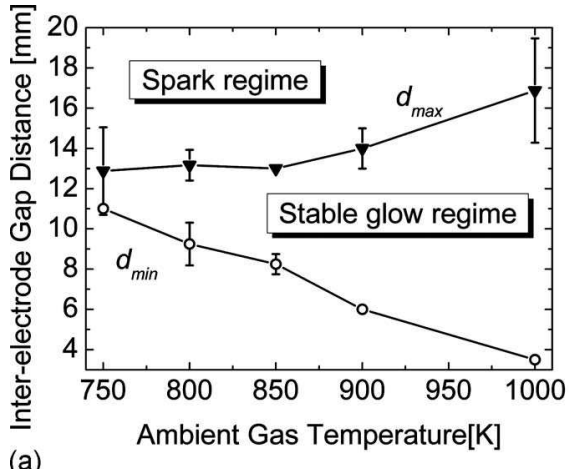

(a)

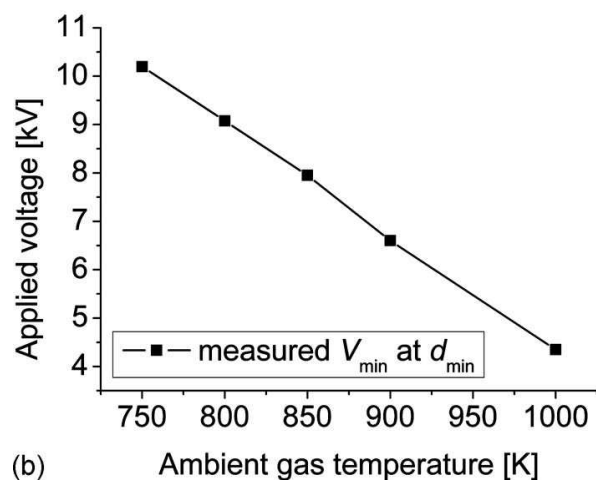

FIG. 10. Measured range of gap distances that permit stable operation of the glow regime (a), as a function of gas temperature and applied voltage at minimum gap distance $\left(V_{\min }\right)$ (b), with $f=30 \mathrm{kHz}$ and $v=1.8 \mathrm{~m} / \mathrm{s}$ fixed.

this behavior occurs because the transition to spark is governed by the avalanche-to-streamer transition (AST) at small $d$ and by the thermal instability at large $d$, and these two mechanisms do not scale with $T_{0}$ in the same way.

\section{Range of gap distances for existence of NRP glow regime}

In Sec. III C, we demonstrated that the NRP glow discharge exists over a range of interelectrode gap distances, with the other experimental parameters fixed apart from the applied voltage. Here we seek to understand how this range of gap distances, bounded by $d_{\min }$ and $d_{\max }$, changes with ambient gas temperature. As mentioned in Sec. II, two highvoltage pulse generators were used to obtain the results shown in this work. All the results shown previously were obtained using the FID Technologies generator model FPG 10-30MS. The results shown in this section, however, were obtained using the FID Technologies generator model FPG $30-100 \mathrm{MC} 4 \mathrm{~K}$ because applied voltages exceeding $10 \mathrm{kV}$ were generally required for generating discharges for $d$ $>1 \mathrm{~cm}$. Figure 10(a) shows the measured gap distance range for the existence of a stable glow regime as a function of the gas temperature.

The minimum gap distance for the existence of a stable glow regime is $d_{\min }=3 \mathrm{~mm}$ at $T_{0}=1000 \mathrm{~K}$ and increases up to $10 \mathrm{~mm}$ at $750 \mathrm{~K}$. The maximum gap distance $d_{\max }$ for stable glow regime existence varies more slowly, from 1.6 $\mathrm{cm}$ at $1000 \mathrm{~K}$ to $1.3 \mathrm{~cm}$ at $750 \mathrm{~K}$. The velocity of the initial streamer of the glow regime was also measured to remain constant at about $10^{8} \mathrm{~cm} / \mathrm{s}$ in this temperature range. This 
observation is consistent with the gas heating of the glow discharge near the G-S transition, which we will discuss in Sec. IV B. In air, at the equivalent gas densities studied here, the streamer velocity scales inversely with gas density. ${ }^{17}$ The proportional increase in ambient gas density from 750 to $1000 \mathrm{~K}$ is $33 \%$, whereas the proportional increase with gas heating taken into account is only $22 \%$, according to Eq. (10). This in turn results in less variation in the streamer velocity than if there were no gas heating. Most importantly, since the applied voltage pulses have a full-width-at-halfmaximum of $15 \mathrm{~ns}$, the pulse width is too short to support streamer propagation across gaps larger than $d_{\max }=1.5 \mathrm{~cm}$. These observations indicate that the maximum gap distance could be limited by the streamer propagation velocity. The values of $d_{\min }$ and $d_{\max }$ converge at $700 \pm 50 \mathrm{~K}$, and no stable glow regime can be obtained at lower temperatures. Based on the foregoing observations, we believe that increasing the pulse duration is one way to obtain the stable NRP glow regime between 300 and $750 \mathrm{~K}$. This simple change is complicated by the fact that increasing the pulse duration also alters the physics of the NRP G-S transition, as will be discussed in Sec. IV B. However, as will be discussed in Sec. IV C, there are other possibilities for achieving stable NRP glow discharges down to $300 \mathrm{~K}$, notably by reducing the electrode radius of curvature.

Figure 10(b) shows that the applied voltage required for generating the NRP glow discharge at $d_{\min }$, called $V_{\min }$, increases linearly with decreasing ambient gas temperature. In Sec. IV C, we will propose an explanation for the value of $d_{\min }$ using the data shown in Fig. 10.

\section{THEORY OF REGIME TRANSITIONS OF NRP DISCHARGES}

In this section we present a theoretical framework for describing the experimental results on NRP discharge transitions from Sec. III, particularly the regime maps. We will demonstrate that the C-G and G-S regime transitions can be well described by the theories of simple dielectric breakdown, the AST, and the thermal ionization instability. From this framework, we will demonstrate that the minimum gap distance for the existence of the NRP glow discharge is related to a new phenomenon that cannot be explained by standard length scales of gas discharges, such as the Paschen minimum and the Meek-Raether criterion for streamer inception.

\section{A. The NRP C-G transition}

To describe this transition, we introduce a simple model of the NRP glow regime. Experimental work ${ }^{11-13}$ has shown that the dynamics of the NRP glow regime can be described by three events. First, an initial cathode-directed streamer creates a conducting channel in the gap. An ensuing return wave of potential redistribution leaves the channel with a plasma structure similar to that of a typical glow discharge: a positive column occupies most of the gap, and a spacecharge layer (formerly the streamer head) situated at the cathode. The final phase is a conduction phase during which current is driven through the discharge channel. This sequence of events is similar to that of the glow phase of the transient arc, as reported in Ref. 18.

We now propose a model to describe the electric field in the streamer and conduction phases that suffices to explain the discharge transitions. For the streamer phase, we concern ourselves only with streamer initiation, and therefore we assume that the electric field at the moment of initiation is well-approximated by the Laplacian electric field $E_{\mathrm{L}}$. For the conduction phase, we assume that the space charge in the discharge channel imposes a cathode fall region and a uniform field in the positive column.

\section{Streamer phase}

The event of interest during the streamer phase is the AST. To analyze the AST, we must first determine the Laplacian electric field $E_{\mathrm{L}}$. For a pin-pin electrode configuration, we approximate the electrodes as two hyperboloid surfaces with foci separated by $a=[(d / 2)(d / 2+R)]^{1 / 2}$, where $d$ is the interelectrode gap distance and $R$ is the radius of curvature of the electrode tip. For our experimental setup, we estimate that $R \approx 200 \mu \mathrm{m}$.

An exact solution exists for the Laplacian field of a pinplane configuration. ${ }^{19,20}$ We use this solution in Appendix B to derive an approximate expression of the Laplacian electric field $\left(E_{\mathrm{L}}\right)$ along the $z$-axis (the interelectrode axis relating the two pins) for the pin-pin case

$$
E_{\mathrm{L}}(z) \approx-\frac{V_{\mathrm{p}}}{\ln \left[\left(1+\eta_{0}\right) /\left(1-\eta_{0}\right)\right]} \frac{a}{a^{2}-z^{2}},
$$

where $V_{\mathrm{p}}$ is the potential of the anode pin, the cathode pin is at zero potential, and $\eta_{0}=[(d / 2) /(d / 2+R)]^{1 / 2}$. Recently, Celestin et $a l^{21,22}$ used a similar Laplacian field expression to simulate the NRP discharges studied here. From Eq. (1), it can be shown numerically that the electric field near the tip is primarily determined by the radius of curvature of the tip, whereas the electric field in the middle of the gap primarily depends on the gap distance. If we widen the gap, $E_{\mathrm{L}}$ near the electrode tips (of fixed radius of curvature $R$ ) decreases less than it does in the middle of the gap.

The AST occurs when the space charge field generated by an electron avalanche becomes comparable with the Laplacian field. ${ }^{23}$ From Ref. 23, the space charge field $E_{\mathrm{SC}}$ generated by an electric dipole formed by two spheres with charges $+Q$ and $-Q$, separated by a distance $\alpha^{-1}$, is given by

$$
E_{\mathrm{SC}}=\frac{2 e Q \alpha^{2}}{9 \pi \varepsilon_{0}} \equiv K \alpha^{2},
$$

where $e$ is the charge of an electron, $Q$ is the number of charges required for the AST (also known as the ionization integral), $\varepsilon_{0}$ is the permittivity of free space, and $\alpha$ is the first Townsend or ionization coefficient. When a uniform external field $E_{\text {unif }}$ is applied, the AST will occur when $E_{\mathrm{SC}}=E_{\text {unif }}$. The above analysis does not apply directly to our case because $E_{\mathrm{L}}$ from Eq. (1) is not uniform.

However, we can still use Eq. (2) by demonstrating that $E_{\mathrm{L}}$ is approximately uniform near the electrode tip located at $z=d / 2$, where we expect the AST to occur because the ion- 


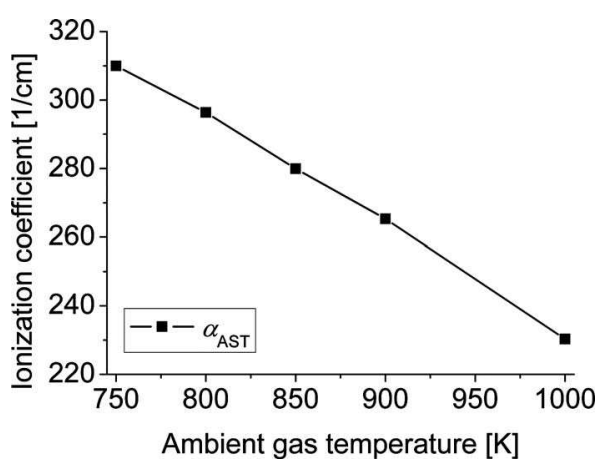

FIG. 11. Calculated ionization coefficient at anode tip for NRP glow discharge at $d_{\min }$ as a function of the ambient gas temperature, using the measured values of $d_{\min }$ and of the applied voltage from Fig. 10.

ization coefficient $\alpha$ depends exponentially on the field, which rises sharply near the electrode tip according to Eq. (1). The Laplacian field at the tip is well above the breakdown field, and in such a situation $Q=\exp (16)$ has been calculated to be an appropriate value of the ionization integral for the AST. ${ }^{24}$ Using the measured applied voltages shown in Fig. 10(b) and the corresponding gap distances from Fig. 10(a), we can calculate the field at the tip using Eq. (1), with $R \approx 200 \mu \mathrm{m}$. Then using Eq. (2) with $E_{\text {unif }}=E_{\mathrm{L}}(z=d / 2)$, it is possible to calculate the ionization coefficient necessary for the AST $\left(\alpha_{\mathrm{AST}}\right)$. Figure 11 shows the values of $\alpha_{\mathrm{AST}}$ obtained as a function of the ambient gas temperature $T_{0}$. In the range $T_{0}=750-1000 \mathrm{~K}, \alpha_{\mathrm{AST}}$ is inversely proportional to $T_{0}$. The smallest value of $\alpha_{\mathrm{AST}}$ is at $T_{0}=1000 \mathrm{~K}$ and corresponds to an ionization length of $43 \mu \mathrm{m}$, which is significantly smaller than $R$. Thus, over the ionization length, the field is effectively uniform, which allows us to use Eq. (2).

It should be noted that the measurements from Fig. 10 are for $d=d_{\min }$ but $\alpha_{\mathrm{AST}}$ does not depend on the gap distance because the AST is completely determined by $E_{\text {unif }}$ for a given value of $Q$, as $\alpha$ depends only on the reduced electric field for a given gas and density. Furthermore, the values for $\alpha$ shown in Fig. 11 are lower than those calculated using the empirical Townsend formula $\alpha=A N_{0} \exp \left(-B N_{0} / E_{\mathrm{L}}\right)$ where $N_{0}$ is the ambient gas density and $A=15 \mathrm{~cm}^{-1} \mathrm{Torr}^{-1}$ and $B=365 \mathrm{~V} / \mathrm{cm} /$ Torr are empirical factors for air. ${ }^{16}$ This is because the space charge field generated by ionization reduces the total field in the dipole region. Thus the value of $\alpha$ obtained with Eq. (2) reflects the total field in the dipole region.

Combining Eqs. (1) and (2) by setting $E_{\mathrm{L}}(z=d / 2)$ $=E_{\text {unif }}=E_{\mathrm{SC}}$ and using $\alpha=\alpha_{\mathrm{AST}}$, we obtain the applied voltage necessary for the AST, $V_{\mathrm{AST}}$

$$
\begin{aligned}
V_{\mathrm{AST}} & =K \alpha_{\mathrm{AST}}^{2}\left[\frac{a^{2}-(d / 2)^{2}}{a}\right] \ln \left(\frac{1+\eta_{0}}{1-\eta_{0}}\right) \\
& \approx K \alpha_{\mathrm{AST}}^{2} R \ln \left(\frac{1+\eta_{0}}{1-\eta_{0}}\right),
\end{aligned}
$$

where the last term on the right-hand side is the approximate expression for $V_{\mathrm{AST}}$ in the case of sharp tip electrodes, $R$ $\ll d$. We will see in Sec. IV A 2 that $V_{\mathrm{AST}}$ is not the C-G transition voltage for interelectrode gap distances well above the minimum gap distance for the existence of the NRP glow regime, $d_{\min }$. The AST becomes important for gap distances near or below $d_{\min }$, and Eq. (3) will be used for understanding $d_{\min }$ in Sec. IV C.

\section{Conduction phase}

Following the streamer and return wave phases, the NRP glow discharge enters the conduction phase, in which the remaining energy of the pulse is dissipated resistively in the conducting channel created by the streamer. During the return wave phase, the space charge in the channel adjusts to redistribute the potential, and thus the field becomes approximately uniform. This smoothing of the field represents the formation of an inhomogeneous positive column ${ }^{16}$ and has been simulated for the conditions of the NRP discharges studied in this work. ${ }^{21,22}$ The field in the positive column $E_{\mathrm{pc}}$ is

$$
E_{\mathrm{pc}}=\frac{V_{\mathrm{p}}-V_{\mathrm{cf}}}{d},
$$

where $V_{\mathrm{p}}$ is the applied voltage and $V_{\text {cf }}$ is the cathode fall voltage. Equation (4) has essentially already been introduced in Sec. III C. To maintain the positive column during the conduction phase, we must have $E_{\mathrm{pc}}>E_{\mathrm{br}}$, where $E_{\mathrm{br}}$ is the breakdown field, i.e., the electric field necessary at a given gas temperature to balance the ionization and attachment rates in air.

Note that this is a situation specific to the NRP glow discharge. In a typical dc glow discharge, for which the external field is applied for much longer than ten nanoseconds, electron losses by recombination, attachment, or diffusion are compensated by the influx of electrons generated in the cathode fall region. However, in the case of the NRP glow, there is insufficient time for cathode-generated electrons to drift into the positive column. The discharge would immediately disappear due to electron losses if the field in the positive column were not at least $E_{\mathrm{br}}$, to ensure that the net ionization frequency is above zero.

The choice of breakdown voltage $E_{\mathrm{br}}$ becomes an issue in fitting the data for different temperatures. The reduced field $\left(E_{\mathrm{br}} / N\right)$ for breakdown, defined by the equality of the ionization and attachment coefficients of air, was calculated to remain about constant up to $2000 \mathrm{~K}^{25}$ Therefore, the breakdown field $E_{\mathrm{br}}$ should scale with the gas temperature according to $E_{\mathrm{br}}=E_{0} \delta$, where $E_{0}$ is the breakdown field in standard conditions and $\delta$ is the relative gas density. Figure 12 shows a comparison of the measured $\mathrm{C}-\mathrm{G}$ transition voltages collected from all experiments (including the data from Figs. 5 and 9) and the theoretical values for breakdown calculated from Eq. (4).

Figure 12 demonstrates that the choice of $E_{0}$ $=27 \mathrm{kV} / \mathrm{cm}$ fits all of the data reasonably well. The variable is the choice of cathode fall voltage $V_{\text {cf }}$, which is found to be $1 \mathrm{kV}$ at $800 \mathrm{~K}$ and $1.8 \mathrm{kV}$ at $1000 \mathrm{~K}$. It can be seen from Fig. 12(a) that for $d=5 \mathrm{~mm}$, the choice of $V_{\mathrm{cf}}=1 \mathrm{kV}$ fits all data points except at $1000 \mathrm{~K}$. Interestingly, these values of $V_{\text {cf }}$ agree roughly with the simulated value of $1.4 \mathrm{kV}$ from a one-dimensional (1D) model of NRP discharges in air at atmospheric pressure at $2000 \mathrm{~K},{ }^{7}$ in which 2 ns pulses at $f$ 

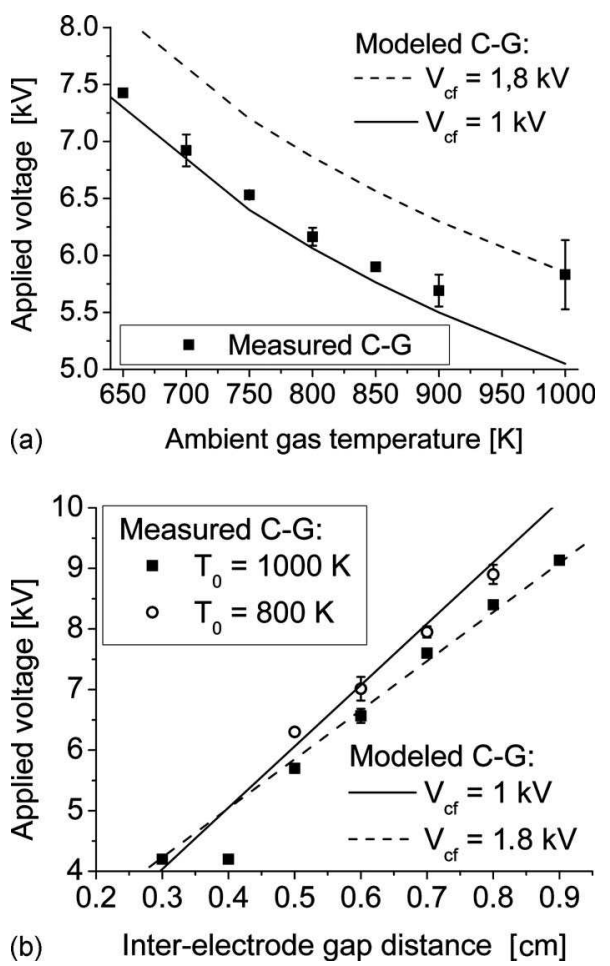

FIG. 12. Comparison of experimental (symbols) and modeled (lines) C-G transition voltage (a) as a function of the ambient gas temperature and (b) of the interelectrode gap distance. The theoretical curves were calculated using $E_{0}=27 \mathrm{kV} / \mathrm{cm}$. The experimental data are taken from Fig. 5 for (a) and Fig. 9 (a) for (b).

$=90 \mathrm{kHz}$ are applied across plane electrodes. Furthermore, these values of $V_{\text {cf }}$ exceed the typical values of $180-370 \mathrm{~V}$ for normal glow discharges in air, ${ }^{16}$ which implies that the NRP glow could be considered an abnormal glow discharge. As mentioned in Sec. II A, the conduction current of the NRP glow is below the detection limit, and so it is not possible to measure the current density directly. However, from Figs. 4 and 7, we see that increasing the applied voltage by about $10 \%$ while operating in the glow regime generally results in an increase in energy per pulse by about an order of magnitude. This implies that the energy increase is largely due to an increase in current. Furthermore, the cathode surface area covered by the glow does not vary appreciably throughout the NRP glow regime [see Figs. 1(c)-1(e) from Ref. 13]. Thus, the current density of the NRP glow increases with increasing voltage, which is further indication of abnormal glow discharge behavior.

\section{3. $C$ - G regime transition voltage}

From Secs. IV A 1 and IV A 2, we see that to achieve the NRP C-G transition, the applied field must be high enough to perform two tasks: initiate the streamer (AST) and maintain the plasma during the conduction phase. Figure 13 shows a comparison of the measured C-G transition voltages at $1000 \mathrm{~K}$ presented in Sec. III C with theoretical values of breakdown and AST voltages, calculated using Eqs. (3) and (4). We use $\alpha_{\mathrm{AST}}=230 \mathrm{~cm}^{-1}$ at $T_{0}=1000 \mathrm{~K}$, taken from Fig. 11. We see that for most of the range of gap distances investigated, it is the breakdown condition during the conduction phase that determines the $\mathrm{C}-\mathrm{G}$ transition voltage, as it is

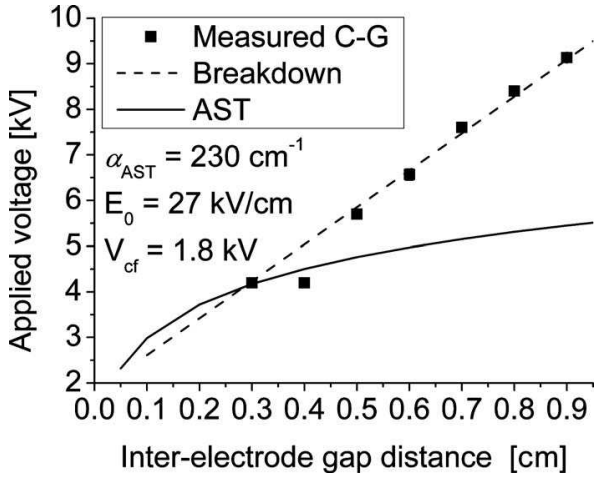

FIG. 13. Measured C-G transition voltage at $1000 \mathrm{~K}$ (symbols) and theoretical values for breakdown and the AST (lines). The measured voltages are the same as the data presented in Figs. 8 and 9.

typically the higher voltage. This is because the pin electrodes create the high local fields required to achieve the AST, whereas the conduction phase has uniform field, thus requiring higher voltage to reach the breakdown condition.

Although Fig. 13 appears to show that only the breakdown condition is necessary to model the $\mathrm{C}-\mathrm{G}$ transition, the importance of the AST in describing the NRP glow will become apparent in Sec. IV C during the discussion of the minimum gap distance for the NRP glow regime.

\section{B. The NRP G-S transition}

Here we will demonstrate that the thermal ionization instability can explain the NRP G-S transition. We base this analysis on the theoretical framework found in standard texts, ${ }^{16,23,26}$ in which gas heating and ionization couple to each other in a continuous fashion. The standard treatment of the G-S transition applies to the case of a dc electric field, as well as to the case of a pulsed field at low repetition frequency in flow. In the latter case, the heated gas is removed from the discharge gap by the flow, and therefore the G-S transition must be induced by a single pulse. Indeed, the G-S transition in atmospheric pressure air has been studied for these cases, ${ }^{18,27}$ and the theoretical analysis of this problem using the framework from Refs. 16, 23, and 26 was used in Ref. 27.

For NRP discharges, however, the high pulse repetition frequency results in incremental heating over a sequence of pulses for as long as the gas remains in the discharge gap. Ionization occurs during the pulse, whereas the period of heating occurs at later times and involves a number of molecular processes, notably vibrational-translational relaxation for air. The NRP G-S transition is thus qualitatively different from those previously studied.

It has been experimentally observed that the NRP G-S transition occurs after a number of pulses ${ }^{11}$ indicating that the NRP G-S transition is a relatively slow process at the millisecond time scale. It has been demonstrated that in air the thermal mechanism for the NRP G-S transition occurs at the microsecond scale, and the chemical kinetic mechanism occurs at the nanosecond scale. ${ }^{28}$ Ref. 28 considers a continuous electric field, and it is reasonable that a pulsed electric field with low duty cycle would result in the transition occurring at the millisecond time scale. Therefore we con- 
clude that in the case of NRP discharges the thermal ionization instability is the cause for the G-S transition.

We can simplify the analysis by considering the NRP heating process to be thermodynamically equivalent to a continuous heating process with the same time-averaged volumetric power. The pulsed volumetric power dissipation is $\sigma E^{2}$, where $\sigma$ is the electrical conductivity and $E$ is the electric field. The time-averaged volumetric power is therefore $\sigma E^{2} \tau f$, where $\tau$ is the time of the conduction phase of the NRP glow discharge described in Sec. IV A 2, and $f$ is the pulse repetition frequency. It has been found that $\tau$ $=5 \mathrm{~ns}$ for the NRP glow discharges described in this work. ${ }^{12}$ The thermal instability therefore occurs when the timeaveraged volumetric power input exceeds the heat loss rate due to conduction and convection

$$
\sigma E^{2} \tau f=N c_{\mathrm{p}}\left(T-T_{0}\right) \nu_{\mathrm{h}}
$$

where $N$ is the gas density in the plasma, $c_{\mathrm{p}}$ is the specific heat per molecule at constant pressure, $T$ is the gas temperature in the plasma, $T_{0}$ is the ambient gas temperature, and $\nu_{\mathrm{h}}$ is the heat removal frequency. The heat removal frequency is the sum of the heat removal frequencies by conduction $\left(\nu_{\text {cond }}\right)$ and by convection $\left(\nu_{\text {conv }}\right)$ (Refs. 16 and 26)

$$
\nu_{\mathrm{h}}=\nu_{\text {cond }}+\nu_{\text {conv }}=\frac{8 \bar{\lambda}}{N c_{\mathrm{p}} r_{t}^{2}}+\frac{2 v}{d},
$$

where $\bar{\lambda}$ is the mean thermal conductivity of the air plasma, $r_{t}$ is the thermal radius of the plasma, $v$ is the airflow velocity, and $d$ is the gap distance. The thermal radius and the electrical radius $\left(r_{\mathrm{p}}\right)$ are defined as the distances from the axis at which $T(r)-T_{0}$ (where $r$ is the radial coordinate) and the electrical number density, respectively, drop to $1 / e$ of their axial values. It has been demonstrated that the ratio $r_{t} / r_{\mathrm{p}}$ remains about constant at values of 2-3 for NRP spark discharges in a quasistationary state, ${ }^{29}$ and we assume that the same is true for the NRP glow regime. For the experiments discussed in this article, we have $v \sim 1 \mathrm{~m} / \mathrm{s}, d$ $\sim 1 \mathrm{~cm}$, and $N \sim 10^{19} \mathrm{~cm}^{-3}$. From ICCD camera images, it has been determined that for the NRP glow, the emission radius is on the order of $100 \mu \mathrm{m}$. Assuming that the emission radius is a reasonable approximation of $r_{\mathrm{p}}$, we see that for these conditions $\nu_{\text {cond }} \sim 10 \nu_{\text {conv }}$, and therefore we can make the approximation $\nu_{\mathrm{h}} \approx \nu_{\text {cond }}$.

From Fig. 7, we observe that the maximum energy deposited per pulse, $U \approx 10 \mu \mathrm{J}$, of the NRP glow discharge does not vary significantly with the ambient gas temperature $T_{0}$ in the range $700-1000 \mathrm{~K}$. The volumetric input power in terms of $U$ is

$$
\sigma E^{2} \tau=\frac{U}{\pi r_{\mathrm{p}}^{2} d} .
$$

Using Eqs. (6) and (7), we can rewrite the energy balance Eq. (5) as

$$
\bar{\lambda}\left(T-T_{0}\right) \simeq U_{l} f / \pi \equiv \Theta,
$$

where $U_{l}=U / d$ is the input energy per pulse and per unit length of the discharge, and $\pi \Theta$ is the input power per unit length. The difference in gas temperature between the plasma and the ambient air is inversely proportional to the average thermal conductivity, because $\Theta$ is a constant under given experimental parameters $f$ and $d$. For the same experimental conditions as in Sec. III B, we measured that $T=1200 \mathrm{~K}$ for $T_{0}=1000 \mathrm{~K},{ }^{12}$ and using $\bar{\lambda}=0.0715 \mathrm{~W} / \mathrm{m} / \mathrm{K}$ for air at the mean temperature of $1100 \mathrm{~K},{ }^{30}$ we have $\Theta=14 \mathrm{~W} / \mathrm{m}$. From 300 to $2000 \mathrm{~K}$, the variation of the thermal conductivity of air with the gas temperature is approximately linear ${ }^{30}$

$$
\lambda=\lambda_{0}+A\left(T-T_{0}\right),
$$

where $\lambda_{0}$ is the thermal conductivity of the ambient gas and $A=6.2 \times 10^{-5} \mathrm{~W} / \mathrm{m} / \mathrm{K}^{2}$. From Eqs. (8) and (9), and taking the average thermal conductivity $\bar{\lambda}=\left(\lambda_{0}+\lambda\right) / 2$, we can deduce the gas temperature increase for the NRP glow discharge just below the G-S transition

$$
T-T_{0}=\frac{-\lambda_{0}+\sqrt{\lambda_{0}^{2}+2 A \Theta}}{A} .
$$

Now we assume that the G-S transition occurs at the same reduced field $(E / N)_{\mathrm{GS}}$ at all ambient gas temperatures between 300 and $1000 \mathrm{~K}$. In Appendix A, we will demonstrate that this assumption is valid for constant $\Theta$, which is the case in our experiments, as mentioned before. A realistic value for $(E / N)_{\mathrm{GS}}$ can be taken from Fig. 5, which shows that $V_{\mathrm{GS}}$ $=6.3 \mathrm{kV}$ at $T_{0}=1000 \mathrm{~K}$. Furthermore, from Fig. 9(b) we see that the cathode fall of the glow discharge just under the G-S transition at $T_{0}=1000 \mathrm{~K}$ is $V_{\text {cf }}=1.9 \mathrm{kV}$. This is deduced by extrapolating the measured G-S transition voltage to $d=0$, much in the same fashion as for the C-G transition as discussed in Sec. IV A 2. In Ref. 12, it was shown that at $T_{0}$ $=1000 \mathrm{~K}$, the NRP glow gas temperature is at $T=1200 \mathrm{~K}$. Thus, the reduced electric field in the positive column of the NRP glow discharge just below the G-S transition at $T_{0}$ $=1000 \mathrm{~K}$ is $144 \mathrm{Td}\left(1 \mathrm{Td}=10^{-17} \mathrm{~cm}^{2}\right)$. The field in the positive column is then $E_{\mathrm{pc}}=(E / N)_{\mathrm{GS}} N$, and it is now possible to derive an expression for the $\mathrm{G}-\mathrm{S}$ transition voltage

$$
V_{\mathrm{GS}}=\left[\left(\frac{E}{N}\right)_{\mathrm{GS}} N\right] d+V_{\mathrm{cf}},
$$

where we use $(E / N)_{\mathrm{GS}}=144 \mathrm{Td}, N$ is calculated using Eq. (10), and we take the cathode fall voltage $V_{\text {cf }}=1.9 \mathrm{kV}$. Figure 14(a) shows a comparison of the measured values of C-S and $\mathrm{G}-\mathrm{S}$ transition voltages as a function of the ambient gas temperature, with $d=5 \mathrm{~mm}$ and $f=30 \mathrm{kHz}$, compiled for all experiments and the theoretical values calculated using Eq. (11). The C-S transition is considered here because the glow discharge should appear momentarily before the thermal instability occurs and induces the G-S transition. The C-S transition therefore includes the G-S transition. The theoretical curve for breakdown without gas expansion via heating [i.e., if we replace $E$ and $N$ by $E_{\mathrm{br}}$ and $N_{0}$ in Eq. (11)], also assuming $V_{\mathrm{cf}}=1.9 \mathrm{kV}$, is also shown as $V_{\mathrm{br}}$ for comparison. Figure 14(b) shows a comparison of the measured values of $V_{\mathrm{GS}}$ as a function of the pulse repetition frequency, with $d$ $=5 \mathrm{~mm}$ and $T_{0}=1000 \mathrm{~K}$, and the theoretical values calculated using Eq. (11).

Figure 14(a) demonstrates that thermal effects must be taken into account to correctly model the G-S transition, and 


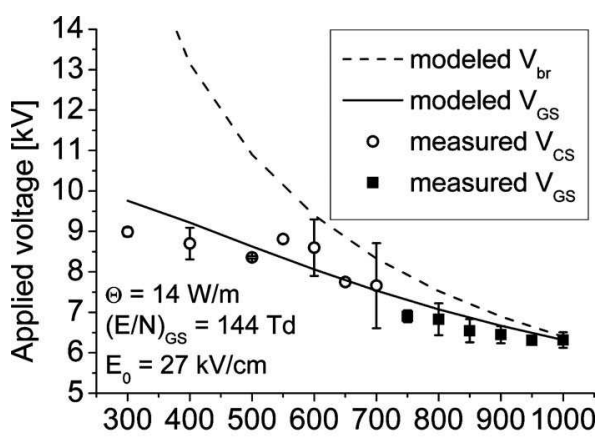

(a) Ambient gas temperature $[\mathrm{K}]$

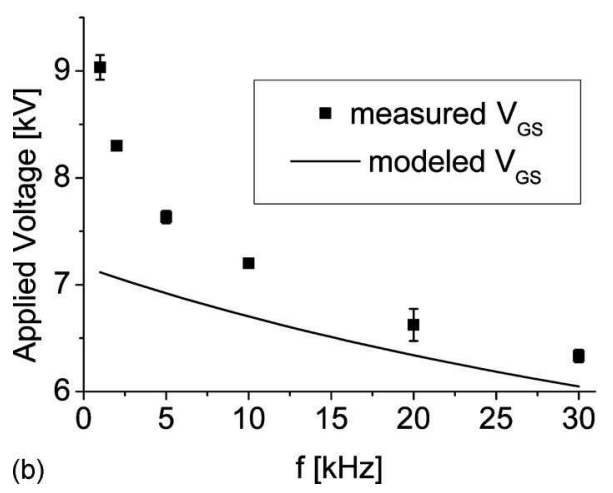

FIG. 14. Comparison of experimental C-S and G-S and modeled G-S transition voltage as a function of (a) ambient gas temperature and (b) pulse repetition frequency. The experimental data shown in (a) are assembled from data shown in Figs. 2, 5, 8, and 9 for the conditions $d=5 \mathrm{~mm}, f$ $=30 \mathrm{kHz}$, and $v=1.6 \mathrm{~m} / \mathrm{s}$. The experimental data shown in (b) are taken from Fig. 2: $T_{0}=1000 \mathrm{~K}, d=5 \mathrm{~mm}$, and $v=1.6 \mathrm{~m} / \mathrm{s}$.

the theoretical curve matches the experimental data quite well. The gas expansion due to discharge-induced heating results in a lower applied voltage for achieving $(E / N)_{\mathrm{GS}}$ than otherwise necessary. Figure 14(b) shows that the theory correctly predicts a rise in $V_{\mathrm{GS}}$ with decreasing pulse repetition frequency, although the rise is not as pronounced as in the experiments. Thus, for the conditions studied experimentally here, the thermal ionization instability adequately describes the G-S transition voltage.

\section{Existence of the NRP glow regime}

The breakdown condition and thermal ionization instability discussed in Secs. IV A 2 and IV B, respectively, suffice to explain the NRP glow and spark regimes at a fixed interelectrode gap distance, as shown in Fig. 5. However, when the gap distance is varied, these two conditions cannot explain the minimum gap distance for the existence of the NRP glow regime. This is demonstrated in Fig. 15, which plots the applied voltages for the AST, breakdown for the C-G transition, and the thermal ionization instability for the G-S transition, as described by Eqs. (3), (4), and (11), respectively. Figure 15 (a) shows that at $T_{0}=1000 \mathrm{~K}$, it is conceivable that $d_{\min }$ is given by the intersection of the applied voltages for the breakdown for the C-G transition, and the thermal ionization instability for the G-S transition. However, Fig. 15(b) demonstrates that at $T_{0}=750 \mathrm{~K}$ only the AST can explain a minimum gap distance.
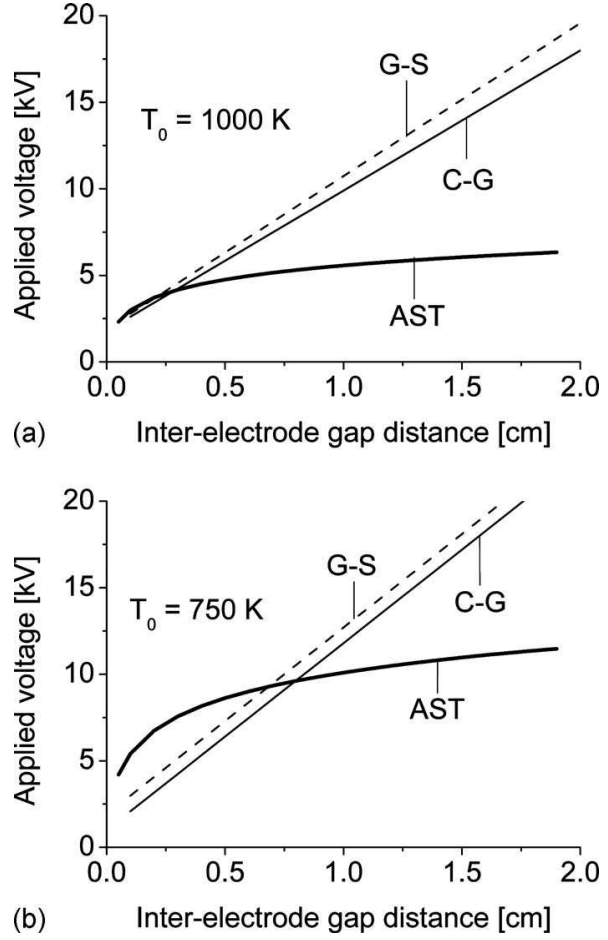

FIG. 15. Theoretical curves at (a) $T_{0}=1000 \mathrm{~K}$ and (b) $750 \mathrm{~K}$ for the applied voltages, as a function of interelectrode gap distance for the AST, breakdown for the C-G transition, and the thermal ionization instability for the $\mathrm{G}-\mathrm{S}$ transition.

Essentially, $d_{\min }$ represents the experimental condition in which the streamer can initiate and yet the heating during the conduction phase does not lead to the thermal ionization instability. For $d<d_{\min }$, the applied voltage is so high that as soon as the streamer crosses the gap, the Joule heating is sufficient to trigger the G-S transition after several pulses. Figure 16 shows that at $d<d_{\min }$, the measured voltages for transition to spark at $T_{0}=1000 \mathrm{~K}$ and $f=30 \mathrm{kHz}$ indeed follow the theoretical curve for the AST instead of that for the thermal ionization instability for the G-S transition, as $V_{\mathrm{AST}}$ is the higher of the two. For $d<d_{\min }$, only the no dischargeto-spark (N-S) transition is observed experimentally. The $\mathrm{N}-\mathrm{S}$ transition is considered here because the corona and glow discharges should appear momentarily before the ther-

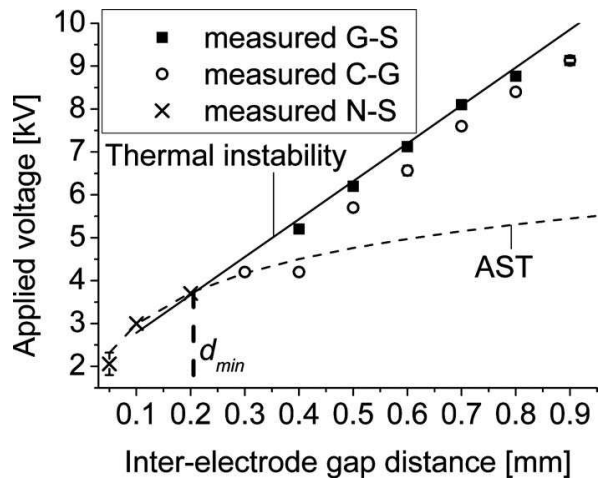

FIG. 16. Comparison of experimental N-S, C-G, and G-S transition voltage and modeled thermal instability threshold and AST, as a function of interelectrode gap distance. $T_{0}=1000 \mathrm{~K}$ and $f=30 \mathrm{kHz}$. The experimental data are taken from Fig. 8. 


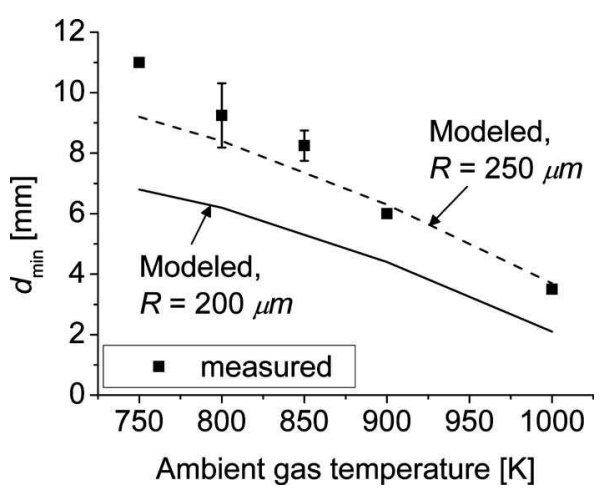

FIG. 17. Comparison between measured and modeled $d_{\min }$ as a function of the ambient gas temperature. $f=30 \mathrm{kHz}$. The experimental data are taken from Fig. 10(a).

mal instability occurs and induces the G-S transition. The $\mathrm{N}-\mathrm{S}$ transition therefore includes the G-S transition. Furthermore, Fig. 9(b) shows that for small gap distances at 400 and $800 \mathrm{~K}$, the measured voltages for transition to spark follow the curved profile of $V_{\mathrm{AST}}$ as a function of gap distance instead of the straight line corresponding to the thermal ionization instability for the G-S transition.

By setting $V_{\mathrm{AST}} \leq V_{\mathrm{GS}}$ and using Eqs. (3) and (11), we derive a criterion for the existence of the NRP glow regime in terms of experimental parameters

$$
\alpha_{\mathrm{AST}}^{2} K R \ln \left[\frac{1+\eta_{0}(d)}{1-\eta_{0}(d)}\right] \leq\left(\frac{E}{N}\right)_{\mathrm{GS}} N d+V_{\mathrm{cf}} .
$$

As is the case with Eq. (11), the right side of Eq. (12) is expressed in terms of the constant value $(E / N)_{\mathrm{GS}}=144 \mathrm{Td}$ and $N$, which is calculated using Eq. (10). Although the pulse repetition frequency is not explicit in Eq. (12), the calculation of the gas density $N$ in the plasma involves $f$ via Eqs. (8) and (10). Furthermore, the pulse duration is indirectly involved in Eq. (12) via Eqs. (5) and (7).

Using the values of $\alpha_{\mathrm{AST}}$ from Fig. 11, it is possible to calculate theoretical values for $d_{\min }$, as a function of the gas temperature using Eq. (12) on the condition of equality. Figure 17 shows a comparison between the theoretical calculation using Eq. (12) and the experimental results from Fig. 10. There is reasonable agreement on the degree of increase in $d_{\text {min }}$ with decreasing ambient gas temperature, assuming an electrode radius of curvature $R=200 \mu \mathrm{m}$. This value is used in calculating the modeled AST transition voltage shown in Figs. 13 and 16, where good agreement with experimental data is found for $V_{\mathrm{AST}}$. However, by assuming $R=250 \mu \mathrm{m}$ instead, better agreement between Eq. (12) and the experimental data is found for $d_{\min }$, as shown in Fig. 17.

It is important to point out that $d_{\text {min }}$ cannot be explained by other length scales common in gas discharge physics. For example, the minimum $p d$ product of the Paschen curve defines the size of a normal cathode fall for a glow discharge at a given pressure (or density in the case of constant pressure but variable gas temperature), and therefore the gap distance must be above the value defined by this minimum $p d$ product. However, we see that this distance decreases with decreasing ambient gas temperature, which is the opposite of the trend shown in Fig. 17. In any case, the measured values for $d_{\text {min }}$ well exceed the typical size of cathode fall regions at high pressure. ${ }^{16}$ Also, the Meek-Raether criterion ${ }^{31}$ for streamer inception (i.e., the AST), commonly expressed as $\alpha d=\ln Q$, implies a minimum gap distance necessary for the avalanche to generate $Q$ electrons by electron-impact ionization. However, we see from Fig. 11 that $\alpha$ increases with decreasing ambient gas temperature. This also implies that $d_{\text {min }}$ would decrease with decreasing ambient gas temperature, which contradicts experiment.

It is worth mentioning that, in gliding arc discharges, there exists a critical length $\left(l_{\text {crit }}\right)$ for the equilibrium (i.e., arc) to nonequilibrium (i.e., glow) transition. ${ }^{32}$ The energy balance between the electrical energy deposited into the discharge and the heat loss from the plasma column plays a role in determining both $l_{\text {crit }}$ and $d_{\text {min }}$ discussed here. However, $l_{\text {crit }}$ differs from $d_{\text {min }}$ because the NRP G-S transition studied here is from the glow to the spark regime, whereas the gliding arc G-S transition is in the opposite direction. As the gliding arc is initially thermal, streamers are not involved in the generation of the glow phase. On the other hand, streamers initiate the NRP glow, making the AST essential to defining $d_{\text {min }}$. Therefore $d_{\min }$ for NRP glows is of a different nature than $l_{\text {crit }}$ for gliding arcs.

Thus, the criterion for the existence of the NRP glow regime described by Eq. (12) is a new development and is unique to NRP discharges. This is because enough heat dissipation occurs between pulses such that the G-S transition can occur at high applied voltages, above those required for the AST. In other types of discharges at atmospheric pressure, notably dc discharges, significant heating occurs at relatively low applied voltage and thus other means must be employed to impede the ionization instability. Such techniques include adding ballast resistors into the discharge circuit or dielectric layers on the electrodes.

Most importantly, Eq. (12) points the way to modifying the experiment to generate the NRP glow regime. For instance, the experimental setup described in this work is only capable of generating the glow discharge down to $750 \mathrm{~K}$. By considering Eq. (12), we see that if the electrodes are made sharper, i.e., by reducing $R$, it is possible to obtain the NRP glow at lower temperatures. This is because by sharpening the electrodes, we lower the voltage necessary to achieve the AST and thereby lower the value of $d_{\min }$. If $d_{\min }$ is less than $d_{\text {max }}$ defined by the maximum streamer propagation distance, then we can generate the NRP glow regime. If sharpening the electrodes is not an option, then it may be possible to increase $d_{\max }$ by increasing the pulse duration.

\section{CONCLUSION}

In this article, we have demonstrated that the corona, glow, and spark regimes of NRP discharges exist in air at atmospheric pressure, in the temperature range of 300 to $1000 \mathrm{~K}$. Experiments have been performed to determine the discharge "regimes," where a regime of a type of discharge is the applied voltage range over which it occurs, with all other experimental conditions fixed. Experiments have been performed to determine the dependence of the regimes on gas temperature, pulse repetition frequency, and interelectrode 
gap distance. Our principal objective was to determine how these parameters affected the NRP glow regime, because we wish to extend its use down to $300 \mathrm{~K}$.

Experimentally, we found that the corona and spark discharge regimes were easily obtained under almost all experimental conditions, whereas the glow regime often existed only over a limited range of the conditions. Using our current experimental setup, we were able to generate the NRP glow discharge from 750 to $1000 \mathrm{~K}$. The most important observation concerning the existence of the NRP glow regime was the relationship between interelectrode gap distance and ambient gas temperature. When the gap distance is fixed, the glow discharge can only be generated above a minimum gas temperature. Conversely, when the gas temperature is fixed, the glow discharge can only be generated in a range of gap distances. We have suggested that the maximum gap distance is defined by the distance that the initial streamer can travel over the pulse duration. Explaining the minimum gap distance required developing the theory described in Sec. IV.

Other notable experimental results include measurements of the energy deposited per pulse into the discharge. The glow discharge was found to dissipate from 1 to $10 \mu \mathrm{J}$ per pulse, whereas the spark discharge was found to dissipate from 100 to $1000 \mu \mathrm{J}$ per pulse. The most useful observation was that the glow discharges generated at applied voltages just under the G-S transition were found to dissipate about $10 \mu \mathrm{J}$ regardless of the ambient gas temperature. This information was critical for the theory developed subsequently in Sec. IV. Furthermore, we found that the G-S transition became less distinct as the pulse repetition frequency was lowered from 30 down to $1 \mathrm{kHz}$, in terms of the variation of measured light emission.

To explain the experimental results, a theory has been developed for the C-G and G-S transitions. We used the experimental observations from previous work ${ }^{11-13}$ to describe the glow discharge quantitatively in terms of two phases. The first is a streamer phase in which the key event is the AST. To calculate the Laplacian field required for the AST, we approximated the electrodes as hyperboloid sheets. The second phase is a conduction phase, in which the space charge of the newly-generated plasma channel creates a uniform field in the gap, apart from the cathode fall region. To maintain the plasma, we presumed that the field in the channel had to remain above the breakdown threshold. Using this simplified description of the glow discharge agreed well with the measured voltages for the $\mathrm{C}-\mathrm{G}$ transition presented in Sec. III.

We also found that the G-S transition was well-described by the thermal ionization instability. As mentioned before, we found from experiment that the input power per pulse of the glow discharge at an applied voltage just under the G-S transition was about constant. In Appendix A, it is demonstrated that given this condition, the reduced field at the G-S transition is also constant. These observations allowed us to calculate the gas temperature rise in the plasma above the ambient temperature and thereby calculate the applied voltage necessary for the G-S transition.

It was found that the aforementioned minimum gap distance for the existence of the NRP glow regime could be understood by considering that the applied voltage of the AST must be lower than that of the thermal ionization instability. This criterion for the existence of the NRP glow regime is expressed by Eq. (12), which represents, to the authors' knowledge, a previously unknown criterion for generating glow discharges. It is in fact unique to NRP glow discharges, because repetitive pulsing permits significant heat dissipation between pulses, thus increasing the voltage that can be applied before the discharge "overheats" and turns into a spark. In other types of stationary discharges, this scenario does not arise.

The advantage of the analytical model presented here is that the NRP discharge regime transitions are readily calculable from experimental parameters. It is not trivial to choose the experimental parameters to produce the NRP glow discharge, and the simple theory presented in this work provides a way to determine such parameters without first developing a full numerical model of the discharge.

In comparison with other methods, the NRP method is unique in its high ionization efficiency. This enables the production of diffuse nonthermal plasmas with low power budgets and high densities of active species, which are critical parameters for numerous applications.

\section{ACKNOWLEDGMENTS}

The authors thank the Agence Nationale de la Recherche for its support of the IPER (Grant No. ANR-05-BLAN-0090) and PREPA (Grant No. ANR-09-BLAN-0043) projects and the Ministère de l'Enseignement Supérieur et de la Recherche for support of a Chaire d'Excellence.

\section{APPENDIX A: REDUCED ELECTRIC FIELD FOR THE G-S TRANSITION}

In this appendix, we demonstrate the validity of the assumption made in Sec. IV B that if the G-S transition always occurs at a given input power per unit length $\Theta$, then the reduced field for the G-S transition $(E / N)_{\mathrm{GS}}$ is constant with the ambient gas temperature.

First, we assume that the plasma electrical radius $r_{\mathrm{p}}$ (defined in Sec. IV B) during the conduction phase described in Sec. IV A 2 is equal to the radius of the channel established during the streamer phase described in Sec. IV A 1. This assumption is consistent with time-resolved single-shot images of the NRP glow obtained using an ICCD camera, which show that the emission radius of the discharge does not change between the streamer and conduction phases. The diameter of streamers in air varies inversely with the gas density ${ }^{17,33}$

$$
r_{\mathrm{p}} N=r_{\mathrm{p} 0} N_{0}=\text { constant, }
$$

where $N_{0}$ is the ambient gas density, $N$ is the plasma gas density, $r_{\mathrm{p}}$ is the plasma electrical radius at gas density $N$, and $r_{\mathrm{p} 0}$ is the plasma electrical radius in the case of no gas heating. Equations (7), (8), and (A1) lead to the following expression

$$
\sigma\left(\frac{E}{N}\right)^{2}=\frac{\Theta}{\left(r_{\mathrm{p} 0} N_{0}\right)^{2} \tau f}
$$


Because the right-hand side is a constant, we can determine the variation of the reduced field $E / N$ as a function of the ambient gas density $N_{0}$. As in Ref. 16 we use the logarithmic derivative to study the sensitivity of variations

$$
\frac{d \ln \sigma}{d \ln N_{0}}+2 \frac{d \ln (E / N)}{d \ln N_{0}} \equiv(\hat{\sigma}+2) \frac{d \ln (E / N)}{d \ln N_{0}}=0,
$$

where the logarithmic derivative of a quantity $X$ with respect to $E / N$ is denoted by

$$
\hat{X}=\frac{d \ln X}{d \ln (E / N)} \equiv \frac{d \ln X}{d \ln \mathrm{T}_{e}} .
$$

The linear relationship between the reduced field and the electron temperature leads to the equivalence of varying by $E / N$ or by $T_{e}$. The plasma conductivity $\sigma$ is

$$
\sigma=\frac{n_{e} e^{2}}{m_{e} \nu_{m}}
$$

where $n_{e}$ is the electron number density, $e$ is the electron charge, $m_{e}$ is the electron mass, and $\nu_{m}$ is the electron-heavy collision frequency, which can be approximated as

$$
\nu_{m} \simeq N Q_{m} \sqrt{\frac{8 k T_{e}}{\pi m_{e}}},
$$

where $k$ is Boltzmann's constant, $Q_{m}$ is the energy-average cross-section for momentum transfer between electrons and heavy particles. From Ref. $34, Q_{m} \approx 1.5 \times 10^{-15} \mathrm{~cm}^{2}$ is about constant over a significant range of electron temperatures $T_{e}$. The logarithmic derivative of the conductivity can therefore be expressed as

$$
\hat{\sigma}=\hat{n}_{e}-\hat{\nu}_{m} \text {. }
$$

From page 223 of Ref. 16, we have an expression for the electron density when ionization is much faster than gas dynamic processes, which is true in the case of NRP discharges

$$
n_{e}=\nu_{i} / \beta
$$

where $\nu_{i}$ is the ionization frequency and $\beta$ is the attachment rate, which can be assumed to be independent of the reduced field above $E_{0} / N_{0}$, the reduced field for breakdown. The reduced ionization frequency $\widetilde{\nu}_{i}=\nu_{i} / N$ depends explicitly on the reduced field $E / N .{ }^{16}$ The logarithmic derivatives of the collision frequency and the electron density can be calculated from Eqs. (A6) and (A8), respectively, and substitution into Eq. (A7) leads to

$$
\hat{\sigma}=\hat{\widetilde{\nu}}_{i}-1 / 2
$$

Substituting this expression for $\hat{\sigma}$ into Eq. (A3) leads to

$$
\left(\hat{\widetilde{\nu}}_{i}+\frac{3}{2}\right) \frac{d \ln (E / N)}{d \ln N_{0}}=0 .
$$

One way to satisfy Eq. (A10) is for $\hat{\widetilde{\nu}}_{i}=-3 / 2$, meaning that the ionization frequency is inversely related to the reduced field. This is not true for $E / N>E_{0} / N_{0}$, and therefore the only solution to Eq. (A10) is

$$
\frac{d \ln (E / N)}{d \ln N_{0}}=0 \text {. }
$$

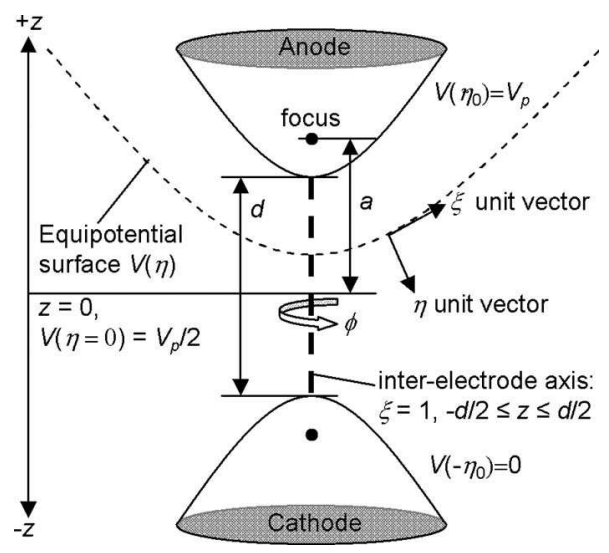

FIG. 18. Two-sheet hyperboloid surface representation of the pin-to-pin electrode configuration in prolate spheroidal coordinates.

Thus, we have proven that if the power input for the G-S transition is independent of the ambient gas density $N_{0}$, then so is the reduced field $E / N$ for the G-S transition.

\section{APPENDIX B: LAPLACIAN ELECTRIC FIELD}

In this appendix we derive the Laplacian electric field generated by a pin-to-pin electrode configuration described by Eq. (1) in Sec. IV A 1. We approximate the electrodes as two hyperboloids because an exact solution to Laplace's equation exists for a pin-plane geometry in prolate spheroidal coordinates $(\eta, \xi, \phi) \cdot{ }^{19,20}$ Hyperboloid surfaces are found when $\eta$ is constant, prolate-spheroidal surfaces are found when $\xi$ is constant, and $\phi$ is the azimuthal angle of rotation about the major axis. Physically, $\eta$-surfaces are equipotential surfaces, and $\xi$ - and $\phi$-lines are electric field lines. As shown in Fig. 18, the origin $(\eta=0, \xi=1)$ is in the center of the discharge gap. We consider the problem in 1D along the interelectrode $(z)$ axis, for which $\xi=1$ and $z=a \eta$, where $2 a$ is the distance between the foci. $R$ is the radius of curvature of the electrode tips, which are located at $z=+d / 2$ for the anode and $z=-d / 2$ for the cathode. $R$ and $d$ are related to the foci distance by $a=[(d / 2)(d / 2+R)]^{1 / 2}$ and to the equipotential surface of the electrodes by $\eta_{0}=[(d / 2) /(d / 2+R)]^{1 / 2} \cdot V\left(\eta_{0}\right)$ $=V_{\mathrm{p}}$ at the anode and $V\left(-\eta_{0}\right)=0$ at the cathode, where $V_{\mathrm{p}}$ is the applied voltage.

For a single hyperboloid electrode at potential $V\left(\eta_{0}\right)$ $=V_{\mathrm{p}}$ facing a plane surface at potential $V(0)=0$ (Ref. 19)

$$
V(\eta)=V_{\mathrm{p}} \frac{\ln [(1+\eta) /(1-\eta)]}{\ln \left[\left(1+\eta_{0}\right) /\left(1-\eta_{0}\right)\right]} .
$$

Because of the cylindrical symmetry of the electrodes, Eq. (B1) is independent of $\xi$ and $\phi$. Recalling that $z=a \eta$, the Laplacian electric field on the interelectrode $(z)$ axis is

$$
E(z)=-\nabla V=-\frac{2 V_{\mathrm{p}}}{\ln \left[\left(1+\eta_{0}\right) /\left(1-\eta_{0}\right)\right]} \frac{a}{a^{2}-z^{2}} .
$$

Equations (B1) and (B2) are also exact solutions for a symmetric pin-to-pin geometry with $V\left(\eta_{0}\right)=V_{\mathrm{p}}$ at the anode, $V(0)=0$ at mid-gap, and $V\left(-\eta_{0}\right)=-V_{\mathrm{p}}$ at the cathode, according to the method of images. Our case shown in Fig. 18 has a different potential distribution, with $V\left(\eta_{0}\right)=V_{\mathrm{p}}$ and 
$V\left(-\eta_{0}\right)=0$. Because $d$ is small compared to the distance between the electrodes and other conducting surfaces at $V=0$ in our experiments, symmetry is nearly preserved, with $V(0)$ $\approx V_{\mathrm{p}} / 2$ and $E(z) \approx-E(-z)$. To account for $V\left(\eta_{0}\right)=V_{\mathrm{p}}, V(0)$ $\approx V_{\mathrm{p}} / 2$, and $V\left(-\eta_{0}\right)=0$, we divide the right side of Eq. (B2) by two to yield Eq. (1) for the Laplacian electric field in our pin-pin electrode configuration.

${ }^{1}$ A. V. Likhanskii, M. N. Shneider, S. O. Macheret, and R. B. Miles, J. Appl. Phys. 103, 053305 (2008).

${ }^{2}$ F. Massines, P. Ségur, N. Gherardi, C. Khamphan, and A. Ricard, Surf. Coat. Technol. 174-175, 8 (2003).

${ }^{3}$ G. Pilla, D. Galley, D. A. Lacoste, F. Lacas, D. Veynante, and C. O. Laux, IEEE Trans. Plasma Sci. 34, 2471 (2006).

${ }^{4}$ A. Schutze, J. Y. Jeong, S. E. Babayan, J. Park, G. S. Selwyn, and R. F. Hicks, IEEE Trans. Plasma Sci. 26, 1685 (1998).

${ }^{5}$ E. Stoffels, I. E. Kieft, R. E. J. Sladek, L. J. M. van den Bedem, E. P. van der Laan, and M. Steinbuch, European Summer School on Low Temperature Plasma Physics (IOP Publishing Ltd., Bad Honnef, Germany, 2004), p. S169.

${ }^{6}$ L. Yu, C. O. Laux, D. M. Packan, and C. H. Kruger, J. Appl. Phys. 91, 2678 (2002).

${ }^{7}$ S. O. Macheret, M. N. Shneider, and R. B. Miles, IEEE Trans. Plasma Sci. 30, 1301 (2002).

${ }^{8}$ C. H. Kruger, C. O. Laux, L. Yu, D. M. Packan, and L. Pierrot, 15 th International Symposium on Plasma Chemistry (International Union of Pure Applied Chemistry, Orleans, France, 2001), p. 337.

${ }^{9}$ M. Nagulapally, G. V. Candler, C. O. Laux, L. Yu, D. M. Packan, C. H. Kruger, R. Stark, and K. H. Schoenbach, 31st AIAA Plasmadynamics and Lasers Conference (Denver, Colorado, 2000), AIAA Paper 2000-2417.

${ }^{10}$ D. M. Packan, Ph.D. thesis, Stanford University, 2003.

${ }^{11}$ D. Pai, Ph.D. thesis, Ecole Centrale Paris, 2008.

${ }^{12}$ D. Z. Pai, G. D. Stancu, D. A. Lacoste, and C. O. Laux, Plasma Sources
Sci. Technol. 18, 045030 (2009).

${ }^{13}$ D. Pai, D. A. Lacoste, and C. O. Laux, IEEE Trans. Plasma Sci. 36, 974 (2008).

${ }^{14}$ I. D. Chalmers, J. Phys. D 4, 1147 (1971).

${ }^{15}$ K. Takaki, D. Kitamura, and T. Fujiwara, J. Phys. D 33, 1369 (2000),

${ }^{16}$ Y. P. Raizer, Gas Discharge Physics (Springer, Berlin, 1991).

${ }^{17}$ S. Pancheshnyi, M. Nudnova, and A. Starikovskii, Phys. Rev. E 71, 016407 (2005).

${ }^{18}$ E. Marode, J. Appl. Phys. 46, 2005 (1975).

${ }^{19}$ G. Mesa, E. DobadoFuentes, and J. J. Saénz, J. Appl. Phys. 79, 39 (1996).

${ }^{20}$ J. D. Zuber, K. L. Jensen, and T. E. Sullivan, J. Appl. Phys. 91, 9379 (2002).

${ }^{21}$ S. Celestin, Z. Bonaventura, B. Zeghondy, A. Bourdon, and P. Ségur, J. Phys. D 42, 065203 (2009).

${ }^{22}$ S. Celestin, Ph.D. thesis, Ecole Centrale Paris, 2008.

${ }^{23}$ E. M. Bazelyan and Y. P. Raizer, Spark Discharge (CRC, Boca Raton, FL, 1998).

${ }^{24}$ C. Montijn and U. Ebert, J. Phys. D 39, 2979 (2006).

${ }^{25}$ Y. Tanaka, J. Phys. D 37, 851 (2004).

${ }^{26}$ A. Fridman and L. A. Kennedy, Plasma Physics and Engineering (CRC Press, New York, 2004).

${ }^{27}$ Y. Akishev, O. Goossens, T. Callebaut, C. Leys, A. Napartovich, and N. Trushkin, J. Phys. D 34, 2875 (2001).

${ }^{28}$ G. V. Naidis, J. Phys. D 38, 3889 (2005).

${ }^{29}$ G. V. Naidis, J. Phys. D 41, 234017 (2008).

${ }^{30}$ J. Taine, E. Iacona, and J.-P. Petit, Transferts Thermiques (Dunod, Paris, 2008).

${ }^{31}$ J. M. A. Meek, Phys. Rev. 57, 722 (1940).

${ }^{32}$ I. V. Kuznetsova, N. Y. Kalashnikov, A. F. Gutsol, A. A. Fridman, and L. A. Kennedy, J. Appl. Phys. 92, 4231 (2002).

${ }^{33}$ T. M. P. Briels, E. M. van Veldhuizen, and U. Ebert, J. Phys. D 41, 14 (2008).

${ }^{34}$ K. H. Becker, U. Kogelschatz, K. H. Schoenbach, and R. J. Barker, Nonequilibrium Air Plasmas at Atmospheric Pressure (IOP Publishing, Bristol, 2005). 